\title{
A GENERALIZATION OF THE STEENROD CLASSIFICATION THEOREM TO $H$-SPACES
}

\author{
BY \\ BYRON DRACHMAN
}

1. Introduction. The purpose of this paper is to show that the Steenrod classification theorem has the following generalization: If $F$ is an associative countable CW $H$-space with cellular multiplication then $B_{F}$, the Dold-Lashof classifying space, classifies principal $F$-bundles. That is, if $X$ is a countable $C W$-complex then there is a one-to-one correspondence between equivalence classes of principal $F$-bundles over $X$ and homotopy classes of maps from $X$ to $B_{F}$.

Since we are working with $H$-spaces instead of groups, we cannot expect an $F$-bundle to be locally trivial. Hence we modify the definition of $F$-bundle so that we have local triviality up to principal homotopy equivalence. Then we do not have the bundle lifting property, but base maps can be lifted so that their projections are homotopic to the base maps. Hence we have to modify the definition of equivalence, namely that the principal maps giving the equivalence do not lie over the identity maps, but rather lie over maps homotopic to the identity maps.

One approach to the Steenrod Classification Theorem considers homomorphisms of $X$ into $G$ (Curtis-Lashof [4]). In our generalization we forsake homomorphisms in favor of strongly homotopy multiplicative maps, which turns out to be the appropriate concept.

The essential step in the proof of this classification theorem is contained in the author's Ph.D. dissertation written under the supervision of Allan Clark, to whom the author is deeply indebted.

The author also wishes to thank James Stasheff for an interesting discussion of this material, and for his generous help in writing the final version of this paper.

\section{Preliminaries.}

$2.1 H$-spaces. By an $H$-space we shall mean a space $X$ with a (continuous) associative multiplication $m: X \times X \rightarrow X$ and a unit element $e \in X$ so that

$$
m(x, e)=m(e, x)=x \text { for all } x \in X
$$

By a CW $H$-space we shall mean an $H$-space $X$ that is a countable CW-complex with cellular multiplication. 
2.2 Quasifiberings. A map $p: E \rightarrow X$ (onto) is defined [15] to be a quasifibering if

$$
p_{*}: \pi_{i}\left(E, p^{-1}(x), y\right) \approx \pi_{i}(X, x) \text { for all } x \in X, y \in p^{-1}(x), i \geqq 0 .
$$

Sugawara has proved the Almost Covering Homotopy Extension Property (ACHEP) for a quasifibering $p: E \rightarrow X$ with fiber $F$ over the base point $*$ of $X$ : Let $(K, L)$ be a CW-pair, $M$ a subcomplex of $K \times I$, and $M^{\prime}=M \cap(K \times(0) \cup L \times I)$. Suppose the following diagram is homotopy commutative

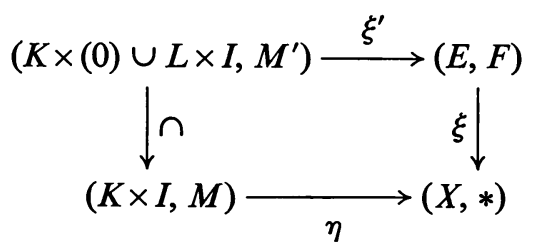

that is $y: p \xi^{\prime} \simeq \eta \mid\left(K \times(0) \cup L \times I, M^{\prime}\right)$ and $y\left(I \times M^{\prime}\right) \subset(*)$. Then the map $\xi^{\prime}$ has an extension $\xi$ such that $Y: p \xi \simeq \eta$ is a homotopy extending $y$, and $Y(I \times M) \subset(*)$.

$F \rightarrow E \rightarrow X$ is a principal quasifibering if (1) $E \rightarrow X$ is a quasifibering with fiber $F$ over the base point and if $F$ is an associative connected $H$-space and $F$ acts associatively on $E$ extending the multiplication in $F$. We assume (see [5, (2.2)]) (2) $\{y\} \cdot H \subset F_{y}=p^{-1}(p(y))$ and (3) $U_{y}: F \rightarrow F_{y}, U_{y}(h)=y \cdot h$ induces $U_{y^{*}}: \pi_{i}(F) \approx \pi_{i}\left(F_{y}\right)$ for all $y$ and $i \geqq 0$.

If $F \rightarrow E_{1} \rightarrow X_{1}$ and $F \rightarrow E_{2} \rightarrow X_{2}$ are two principal quasifiberings, a principal map between the two principal quasifiberings is defined to be a fiberwise map $f: E_{1} \rightarrow E_{2}$ which satisfies $f(y h)=f(y) \cdot h$ for $y \in E, h \in F$. A principal homotopy between the two principal quasifiberings is a map $H: E \times I \rightarrow E_{1}$ so that

$$
H_{t}: E \rightarrow E_{1} \text { is a principal map for all } t \in I \text {. }
$$

Two principal quasifibrations $F \rightarrow E_{1} \rightarrow X$ and $F \rightarrow E_{2} \rightarrow X$ are equivalent if there exist principal maps $f: E_{1} \rightarrow E_{2}$ and $g: E_{2} \rightarrow E_{1}$ lying over maps homotopic to the identity map of $X$ such that $f g$ and $g f$ are both homotopic to the identity maps, the homotopies being given by principal homotopies which lie over maps homotopic to the identity maps for each value of $t$. This is a modification of the definition of Dold and Lashof [5, p. 292]. For the definition of principal F-bundle, see (3.6).

2.3 The Dold-Lashof construction. Let $X$ be an $H$-space with unit $e$. The principal universal quasifibering $X \rightarrow E_{\infty}(X) \rightarrow B_{\infty}(X)$ for $X$ is defined as follows [5]:

Let $E_{0}(X)=X, B_{0}(X)=(e)$, and $p_{0}: E_{0}(X) \rightarrow B_{0}(X)$ be the projection onto the base point. Then $X$ acts on $E_{0}(X), m: E_{0}(X) \times X \rightarrow E_{0}(X)$, by the multiplication in $X$. Having formed the quasifibering $X \rightarrow E_{n}(X) \stackrel{P_{n}}{\rightarrow} B_{n}(X)$ and an action $m: E_{n}(X) \times X \rightarrow E_{n}(X)$, let $E_{n+1}(X)$ be formed from $E_{n}(X) \times I \times X$ by identifying $\left(y_{n}, t, x\right)$ with $m\left(y_{n}, x\right)=y_{n} \cdot x$ if $t=1$ and with $x$ if $t=0 . E_{n+1}(X)$ is given a 
stronger topology than the identification topology. Namely, if $y_{n}|t| x$ represents the class in $E_{n+1}(X)$, the topology is the strongest such that the coordinate functions

$$
\begin{array}{cl}
t: E_{n+1}(X) \rightarrow I, & y|t| x \rightarrow t, \\
y x: t^{-1}(0,1] \rightarrow E_{n}(X), & y|t| x \rightarrow y x, \\
x: t^{-1}[0,1) \rightarrow X, & y|t| x \rightarrow x, \\
y: t^{-1}(0,1) \rightarrow E_{n}(X), & y|t| x \rightarrow y,
\end{array}
$$

are continuous.

$B_{n+1}(X)$ is constructed from $E_{n}(X) \times I$ by identifying $\left(y_{n}, t\right)$ with $e \in X$ if $t=0$ and with $p\left(y_{n}\right) \in B_{n}(X)$ if $t=1$. Letting $[y \mid t]$ represent the class in $B_{n+1}(X)$, $B_{n+1}(X)$ is given the strongest topology so that the coordinate functions

$$
\begin{array}{rlrl}
t: & B_{n+1}(X) \rightarrow[0,1], & & {[y \mid t] \rightarrow t,} \\
y: t^{-1}(0,1) \rightarrow E_{n}(X), & & {[y \mid t] \rightarrow y,} \\
p(y): t^{-1}(0,1] \rightarrow B_{n}(X), & & {[y \mid t] \rightarrow p(y),}
\end{array}
$$

are continuous.

Points in $E_{n}(X)$ may be written, in the notation of Dold and Lashof, as $x_{0}\left|t_{1}\right| \cdots\left|t_{n}\right| x_{n}$, where $\left(t_{1}, \ldots, t_{n}\right) \in I^{n},\left(x_{0}, \ldots, x_{n}\right) \in X^{n+1}$, and

$$
\begin{aligned}
x_{0}\left|t_{1}\right| \cdots\left|t_{n}\right| x_{n} & =\left|x_{i}\right| t_{i+1}|\cdots| t_{n} \mid x_{n} \quad \text { if } t_{i}=0, \\
& =x_{0}\left|t_{1}\right| x_{1}|\cdots| t_{i-1}\left|x_{i-1} x_{i}\right| t_{i-1}|\cdots| t_{n} \mid x_{n} \text { if } t_{i}=1 .
\end{aligned}
$$

The action $m: E_{n}(X) \times X \rightarrow E_{n}(X)$ is described by

$$
m\left(x_{0}\left|t_{1}\right| \cdots\left|t_{n}\right| x_{n}, x\right)=x_{0}\left|t_{1}\right| \cdots\left|t_{n}\right| x_{n} \cdot x .
$$

Points in $B_{n}(X)$ are written as $\left[x_{0}\left|t_{1}\right| \cdots \mid t_{n}\right]$ and

$$
\begin{aligned}
{\left[x_{0}\left|t_{1}\right| \cdots\left|x_{n-1}\right| t_{n}\right] } & =\left[x_{i}\left|t_{i+1}\right| \cdots \mid t_{n}\right] \text { if } t_{i}=0(i<n), \\
& =e \text { if } t_{n}=0, \\
& =\left[x_{0}\left|t_{1}\right| \cdots\left|t_{i}\right| x_{i-1} x_{i}\left|t_{i+1}\right| \cdots \mid t_{n}\right] \text { if } t_{i}=1(1<n), \\
& =\left[x_{0}\left|t_{1}\right| \cdots \mid t_{n-1}\right] \text { if } t_{n}=1 .
\end{aligned}
$$

$p_{n}: E_{n}(X) \rightarrow B_{n}(X)$ is defined by

$$
p_{n}\left(x_{0}\left|t_{1}\right| \cdots\left|t_{n}\right| x_{n}\right)=\left[x_{0}\left|t_{1}\right| \cdots \mid t_{n}\right] .
$$

Then we have $E_{0}(X) \subset E_{1}(X) \subset \ldots \subset E_{n}(X) \subset \cdots$ and $B_{0}(X) \subset B_{1}(X) \subset \ldots \subset$ $B_{n}(X) \subset \ldots$. Let $E_{\infty}(X)$ and $B_{\infty}(X)$ be the corresponding unions. $B_{\infty}(X)$ is given the limit topology, and $E_{\infty}(X)$ is given a stronger topology so that $p_{\infty}: E_{\infty}(X) \rightarrow$ $B_{\infty}(X)$ (the map induced by the $p_{n}$ ) is continuous and $X$ acts continuously on $E_{\infty}(X) . p_{n}: E_{n}(X) \rightarrow B_{n}(X)$ is a quasifibration [15] for all $n, 0 \leqq n \leqq \infty . p_{\infty}: E_{\infty}(X)$ $\rightarrow B_{\infty}(X)$ is the principal universal quasifibering for $X$. It is principal since the action $E_{\infty}(X) \times X \rightarrow E_{\infty}(X)$ is associative and extends the multiplication. It is universal since $\pi_{i}\left(E_{\infty}(X)\right)=0$ for all $i$. 
3. Some fiberings and lifting properties.

3.1 Definition [4, p. 238]. A continuous map $p: E \rightarrow B$ (onto $B$ ) is a weak fibering with respect to a class $\mathscr{C}$ of spaces if it has the weak covering homotopy property (WCHP) with respect to $\mathscr{C}$. That is, given the commutative diagram

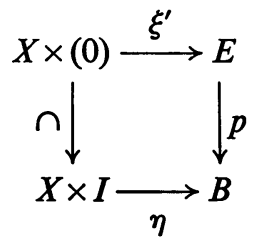

there is a map $\xi: X \times D^{1} \rightarrow E$ such that

$$
\begin{aligned}
p \xi(x, t)=\eta(x, 0) & \text { for }-1 \leqq t \leqq 0, \\
p \xi(x, t)=\eta(x, t) & \text { for } 0 \leqq t \leqq 1, \text { and } \\
\xi(x,-1)=\xi^{\prime}(x, 0) & \left(D^{1}=[-1,1]\right) .
\end{aligned}
$$

In other words, $\eta: X \times I \rightarrow B$ can be lifted to a map $X \times I \rightarrow E$ whose initial position is vertically homotopic to $\xi^{\prime}$. This condition is equivalent to saying that $p: E \rightarrow B$ has the covering homotopy property (with respect to $\mathscr{C}$ ) for all homotopies $\eta: X \times I \rightarrow B$ which are "constant" on $X \times\left[0, \frac{1}{2}\right]$, that is, for which $\eta(x, t)=n(x, 0)$ when $0 \leqq t \leqq \frac{1}{2}$.

3.2 Weak covering homotopy extension property (WCHEP).

Definition. A map $p: E \rightarrow B$ (onto) has the WCHEP with respect to $\mathscr{C}$ if given the pair $(X, A)$ of spaces in $\mathscr{C}$ such that $X \times(0) \cup A \times I$ is also in $\mathscr{C}$ and if given the commutative diagram

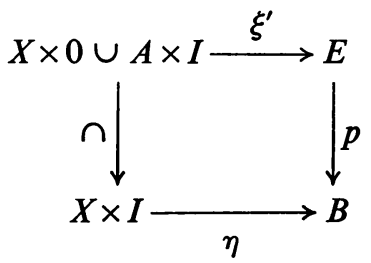

there is a map $\xi: X \times I \rightarrow E$ such that

$$
p \xi=\eta \text { and } \xi \mid X \times 0 \cup A \times I \text { is vertically homotopic to } \xi^{\prime} \text {. }
$$

3.3 TheOREM. If $p: E \rightarrow B$ has the WCHP with respect to $\mathscr{C}$ then $p: E \rightarrow B$ has the WCHEP with respect to $\mathscr{C}$.

Proof. Suppose we are given the commutative diagram

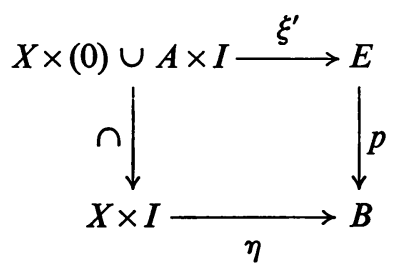


Let $Y$ denote $X \times(0) \cup A \times I$. Define $h: Y \times I \rightarrow B$ as follows:

$$
h(x, u, v)=\eta(x, \max (u, v)) \quad \text { if }(x, u) \in Y \text { and } v \in I .
$$

Then we have the diagram

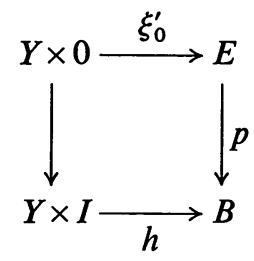

where $\xi_{0}^{\prime}(y, 0)=\xi^{\prime}(y)$. By the WCHP, there is a map $\psi: Y \times D^{1} \rightarrow E$ such that

$$
\begin{aligned}
p \psi(y, t) & =h(y, 0) \text { for }-1 \leqq t \leqq 0, \\
p \psi(y, t) & =h(y, t) \text { for } 0 \leqq t \leqq 1, \text { and } \\
\psi(y,-1) & =\xi_{0}^{\prime}(y,)=\xi^{\prime}(y) .
\end{aligned}
$$

Now define $\xi: X \times I \rightarrow E$ by $\xi(x, t)=\psi(x, 0, t)$ then $p \xi=\eta$ and $\xi \mid X \times(0) \cup A \times I \simeq$ $\xi^{\prime}$. To see this, define a homotopy $H: Y \times I \rightarrow E$ as follows:

$$
\begin{aligned}
H(x, u, t) & =\psi(x, u, 2 t-1) & & \text { if } 0 \leqq t \leqq \frac{1}{2} \\
& =\psi(x, u,(4 t-2) u) & & \text { if } \frac{1}{2} \leqq t \leqq \frac{3}{4} \\
& =\psi(x,(4-4 t) u, u) & & \text { if } \frac{3}{4} \leqq t \leqq 1
\end{aligned}
$$

$H$ is a vertical homotopy, that is $p H(y, t)=\eta(y)$ whenever $y \in Y$.

Hence we see that given

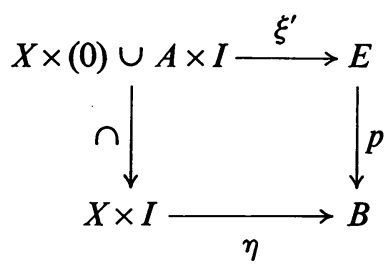

there is a map $\xi: X \times I \rightarrow E$ such that $p \xi=\eta$ and $\xi \mid X \times(0) \cup A \times I$ is vertically homotopic to $\xi^{\prime}$.

If $F \rightarrow E \stackrel{p}{\rightarrow} B$ is a weak fibering, then by Sugawara's result [15] $p: E \rightarrow B$ satisfies the almost covering homotopy property (ACHP) with respect to the category of $\mathrm{CW}$-complexes.

In this paper, we shall be constructing an infinite sequence of maps

$$
\left\{M_{n}: \Omega X \times(I \times \Omega X)^{n} \rightarrow F\right\}
$$

where $F \rightarrow E \rightarrow X$ is a quasifibering and $X$ is a countable CW-complex. Then $\Omega X$ has the homotopy type of a countable $\mathrm{CW}$-complex, but is not one itself in general. Hence we shall modify Sugawara's definition so that it be applicable to the spaces we consider. 
3.4 Definition. The map $p: E \rightarrow B$ (onto) with fiber $F$ has the weak almost covering homotopy extension property (WACHEP) for the pair $(K, L)$ of spaces, if whenever given the homotopy commutative diagram

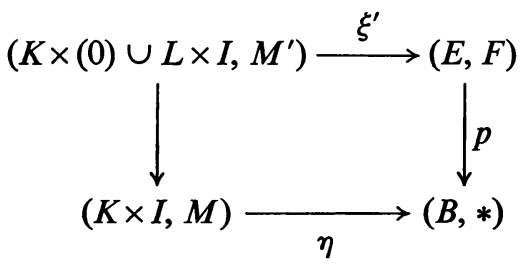

(with $\left.M^{\prime}=(K \times(0) \cup L \times I) \cap M\right)$, that is, there is a homotopy

$$
y:\left(K \times 0 \cup L \times I, M^{\prime}\right) \rightarrow(B, *)
$$

such that $y(, 0)=p \xi^{\prime}$ and $y(, 1)=\eta \mid K \times(0) \cup L \times I$, then $\xi^{\prime}$ has an extension to $\xi$, giving the homotopy commutative diagram

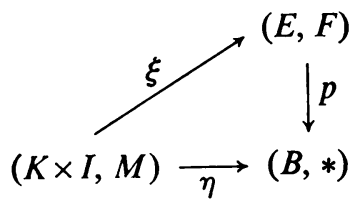

and the homotopy $Y:(K \times I \times I, M \times I) \rightarrow(B, *)$ satisfies

$$
\begin{aligned}
Y(\omega, s, z) & =y(\omega, s, 2 z) & & \text { if }(\omega, s) \in K \times(0) \cup L \times I, 0 \leqq z \leqq \frac{1}{2}, \\
& =\eta(\omega, s) & & \text { if }(\omega, s) \in K \times(0) \cup L \times I, \frac{1}{2} \leqq z \leqq 1 .
\end{aligned}
$$

3.5 THEOREM. Let $F \rightarrow E \stackrel{p}{\rightarrow} X$ be a quasifibering. Suppose $X$ is a countable CW-complex.

Let $K=\Omega X \times(I \times \Omega X)^{n}$ and

$$
L=K \cap\left\{\left(\lambda_{0}, t_{1}, \lambda_{1}, \ldots, t_{n}, \lambda_{n}\right) \mid t_{i} \in \partial I \text { for some } i, 1 \leqq i \leqq n\right\} .
$$

Then $p: E \rightarrow X$ has the WACHEP for $(K, L)$.

For the proof see the appendix.

We now give our modified definition of an $F$-bundle:

3.6 Definition. A principal $F$-bundle is a (almost) locally trivial principal quasifibering $F \rightarrow E \rightarrow X$, i.e.

(LT) for every $\bar{x} \in X$ and neighborhood $W$ of $\bar{x}$ there is a neighborhood $U(\bar{x})$ with $\bar{x} \in U(\bar{x}) \subset W$ and a local cross section $s: U \rightarrow E$ (ps =id) such that the principal map

$$
\psi_{U}: U \times H \rightarrow p^{-1}(U), \quad \psi_{U}(x, h)=s(x) h
$$


is a homotopy equivalence with homotopy inverse $\varphi_{U}: p^{-1}(U) \rightarrow U \times H$ a principal map ( $H$ acts on the second factor of $U \times H)$. We assume



is a commutative diagram where $p_{1}$ is projection on the first factor. We assume also that $\psi_{U} \circ \varphi_{U} \simeq \mathrm{id}$ and $\varphi_{U} \circ \varphi_{U} \simeq \mathrm{id}$, both homotopies being principal homotopies (see 2.2).

4. Strongly homotopy multiplicative maps and induced maps on classifying spaces.

4.1 Definition of $n$-homotopy multiplicative maps of $H$-spaces, and relevant notation.

Notation. The following notation will be useful in dealing with $n$-homotopy multiplicative maps.

Let $X$ be an $H$-space. Let $J=[0, a]$ be an interval. For each integer $n \geqq 0$ define $X_{n}=X \times(J \times X)^{n}$.

Define the maps $t_{i}: X_{n} \rightarrow J, x_{i}: X_{n} \rightarrow X, \delta_{i}: X_{n} \rightarrow X_{n-1},(\alpha, \beta): X_{n} \rightarrow X_{\beta-\alpha}$ $(\alpha \leqq \beta)$ as follows:

If $\omega=\left(x_{0}, t_{i}, x_{i}, \ldots, t_{n}, x_{n}\right) \in X_{n}$, let

$$
\begin{aligned}
t_{i}(\omega) & =t_{i} \quad(1 \leqq i \leqq n), \\
x_{i}(\omega) & =x_{i} \quad(0 \leqq i \leqq n), \\
\delta_{i}(\omega) & =\left(x_{0}, t_{1}, \ldots, t_{i-1}, x_{i-1} x_{i}, t_{i+1}, \ldots, t_{n}, x_{n}\right), \\
\omega(\alpha, \beta) & =(\alpha, \beta)(\omega)=\left(x_{\alpha}, t_{\alpha+1}, \ldots, t_{\beta}, x_{\beta}\right)
\end{aligned}
$$

Definition. Let $Y$ and $Z$ be $H$-spaces. Let $n$ be a positive integer or $+\infty$. An $n$-homotopy multiplicative ( $n$-HM) map from $Y$ to $Z$ is a sequence of maps $\left\{M_{0}, M_{1}, \ldots, M_{n}\right\}$ and each $M_{i}: Y_{n} \rightarrow Z(i=0, \ldots, n)$ satisfies

$$
\begin{aligned}
M_{i}(\omega) & =M_{i-1}\left(\delta_{j}(\omega)\right) \quad \text { if } t_{j}(\omega)=0, \\
& =M_{j-1}\left(\omega_{(0, j-1)}\right) \cdot M_{i-j}\left(\omega_{(j, i)}\right) \quad \text { if } t_{j}(\omega)=a .
\end{aligned}
$$

(In the above $Y_{n}=Y \times(J \times Y)^{n}$ where $J=[0, a]$.)

An $\infty$-HM map from $Y$ to $Z$ is also called a strongly homotopy multiplicative (SHM) map from $Y$ to $Z$. This definition is a modification of the definition given by Sugawara [16, p. 259].

If a map from $Y$ to $Z$ is $1-\mathrm{HM}$, then this corresponds to the usual definition of a homotopy multiplicative map. Also, if $J=[0,0]$ then the map $M_{0}$ is multiplicative.

4.2 Compositions of $n-H M$ maps. 
TheOrem (Clark [3]). Let $H, K$, and $L$ be $H$-spaces. Let $\left\{M_{0}, \ldots, M_{n}\right\}$ be an $n-H M$ map from $H$ to $K$ and $\left\{N_{0}, \ldots, N_{n}\right\}$ be an $n-H M$ map from $K$ to $L$.

Then there is an $n$-HM map $\left\{P_{0}, \ldots, P_{n}\right\}$ from $H$ to $L$.

Proof. We are given maps and intervals

$$
\begin{array}{rlrl}
J_{1} & =[0, a], & J_{2}=[0, b], & \\
M_{i}: H_{i} \rightarrow K, \quad 0 & \leqq i \leqq n, & N_{i}: K_{i} \rightarrow L, \quad 0 \leqq i \leqq n,
\end{array}
$$

where $H_{i}=H \times\left(J_{1} \times H\right)^{i}$ and $K_{i}=K \times\left(J_{2} \times K\right)^{i}, 0 \leqq i \leqq n$. Now let $J_{3}=[0, a+b]$ and $H_{i}^{\prime}=H \times\left(J_{3} \times H\right)^{i}, \quad 0 \leqq i \leqq n$. Define $P_{0}=N_{0} M_{0}$. Now define, for $1 \leqq m \leqq n$, $P_{m}: H_{m}^{\prime} \rightarrow L:$ Given $\omega \in H_{m}^{\prime}$, suppose $a \leqq t_{i}(\omega) \leqq a+b$ whenever $i=j_{1}, \ldots, j_{k}$ and $0 \leqq t_{i}(\omega) \leqq a$ whenever $i \neq j_{1}, \ldots, j_{k}$. Then define

$$
P_{m}(\omega)=N_{k}\left(z_{0}, t_{j_{1}}(\omega)-a, \ldots, t_{j_{k}}(\omega)-a, z_{k}\right)
$$

where

$$
z_{v}=M_{j_{v}+1-j_{v-1}}\left(\omega_{\left(j_{v}, j_{v+1}-1\right)}\right) .
$$

Then using this result, given $\left\{M_{0}, \ldots, M_{n}\right\}$ and $\left\{N_{0}, \ldots, N_{n}\right\}$, we define the composition $\left\{N_{0}, \ldots, N_{n}\right\}\left\{M_{0}, \ldots, M_{n}\right\}$ to be the $\operatorname{map}\left\{P_{0}, \ldots, P_{n}\right\}$.

Fuchs [17] points out that composition of $n$-HM maps is not associative. However, by introducing the interval $J$ instead of the unit interval, we now have that the composition is associative. This is analogous, of course, to choosing the associative Moore loop space, where a loop can have any length, rather than the nonassociative loop space where loops have length 1 .

In order to define homotopies between $n$-HM maps, which can have different lengths, we first normalize $n$-HM maps in the following way:

4.3 Homotopies of $n-H M$ maps.

4.3.1 Definition. Let $H$ and $K$ be $H$-spaces. Let $\left\{M_{0}, \ldots, M_{n}\right\}$ be an $n$-HM map from $H$ to $K$. Then, by definition, for some interval $J=[0, a]$, we are given $M_{i}: H \times(J \times H)^{i} \rightarrow K, i=0, \ldots, n$. Define the normalized $n$-HM map $\left\{\bar{M}_{0}, \ldots, \bar{M}_{n}\right\}$ from $H$ to $K$ by defining, for each $i$,

$$
\begin{gathered}
\bar{M}_{i}: H \times(I \times H)^{i} \rightarrow K \quad(I=\text { unit interval }), \\
\bar{M}_{i}\left(x_{0}, t_{1}, \ldots, t_{i}, x_{i}\right)=M_{i}\left(x_{0}, t_{1} \cdot a, \ldots, t_{i} \cdot a, x_{i}\right) \quad \text { if }\left(x_{0}, t_{1}, \ldots, t_{i}, x_{i}\right) \in H \times(I \times H)^{i} .
\end{gathered}
$$

We can now define homotopies between $n$-HM maps.

4.3.2 Definition. Let $H$ and $K$ be $H$-spaces. Let $\left\{M_{0}, \ldots, M_{n}\right\}$ be an $n$-HM map from $H$ to $K$, and also let $\left\{N_{0}, \ldots, N_{n}\right\}$ be an $n$-HM map from $H$ to $K$. Then $\left\{M_{0}, \ldots, M_{n}\right\}$ and $\left\{N_{0}, \ldots, N_{n}\right\}$ are homotopic if there is a sequence of maps $\left\{h_{0}, \ldots, h_{n}\right\}, h_{i}: I \times H(I \times H)^{i} \rightarrow K$ satisfying, for $t_{0} \in I, \omega \in H \times(I \times H)^{i}$,

$$
\begin{aligned}
h_{i}\left(t_{0}, \omega\right) & =\bar{M}_{i}(\omega) \quad \text { if } t_{0}=0, \\
& =\bar{N}_{i}(\omega) \quad \text { if } t_{0}=1,
\end{aligned}
$$


and also satisfying, for each $t_{0} \in I$, the requirement that $\left\{h_{0}\left(t_{0}\right), \ldots, h_{n}\left(t_{0}\right)\right\}$ is an $n$-HM map from $H$ to $K$, where $h_{i}\left(t_{0}\right): H \times(I \times H)^{i} \rightarrow K$ is defined by $h_{i}\left(t_{0}\right)(\omega)=$ $h_{i}\left(t_{0}, \omega\right)$ when $t_{0} \in I, \omega \in H \times(I \times H)^{i}$.

We shall from now on assume that any SHM map is already normalized, that is, $J$ is chosen to be the unit interval.

4.3.3 Induction of maps on projective spaces. The motivation for the definition of an $n$-HM map is the next theorem.

4.3.4.1 Theorem (Sugawara [16, p. 260]). Let $X$ and $Y$ be H-spaces. Let $M=\left\{M_{0}, \ldots, M_{n}\right\}$ be an $n-H M$ map from $X$ to $Y$. Let $X \rightarrow E_{n}(X) \rightarrow B_{n}(X)$ and $Y \rightarrow E_{n}(Y) \rightarrow B_{n}(Y)$ be the nth stages of the Dold-Lashof constructions of the universal principal quasifibrations for $X$ and $Y$. Then $\left\{M_{0}, \ldots, M_{n}\right\}$ induces maps $E_{n}(M): E_{n}(X) \rightarrow E_{n}(Y)$ and $B_{n}(M): B_{n}(X) \rightarrow B_{n}(Y)$ so that $E_{n}(M) \mid X=M_{0}$ and the diagram

is commutative.

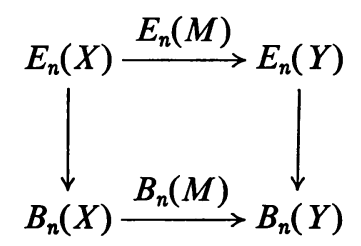

For example, if $n=1$, writing $M_{0}=f$,

$$
\begin{array}{rlrl}
E_{1}(M)\left(x_{0}\left|t_{1}\right| x_{1}\right) & =f\left(x_{0}\right)\left|2 t_{1}\right| f\left(x_{1}\right), & 0 \leqq t_{1} \leqq \frac{1}{2}, \\
& =M_{1}\left(x_{0}, 2-2 t_{1}, x_{1}\right), \quad \frac{1}{2} \leqq t_{1} \leqq 1 ; \\
B_{1}(M)\left[x_{0} \mid t_{1}\right] & =\left[f\left(x_{0}\right) \mid 2 t_{1}\right] & & \text { if } 0 \leqq t_{1} \leqq \frac{1}{2}, \\
& =* & & \text { if } \frac{1}{2} \leqq t \leqq 1 .
\end{array}
$$

To give one more example, if $n=2$,

$$
\begin{aligned}
E_{2}(M)\left(x_{0}\left|t_{1}\right| x_{1}\left|t_{2}\right| x_{2}\right) & =f\left(x_{0}\right)\left|2 t_{1}\right| f\left(x_{1}\right)\left|2 t_{2}\right| f\left(x_{2}\right) \quad \text { if } 0 \leqq t_{1} \leqq \frac{1}{2} \text { and } 0 \leqq t_{2} \leqq \frac{1}{2}, \\
& =M_{1}\left(x_{0}, 2-2 t_{1}, x_{1}\right)\left|2 t_{2}\right| f\left(x_{2}\right) \quad \text { if } \frac{1}{2} \leqq t_{1} \leqq 1 \text { and } 0 \leqq t_{2} \leqq \frac{1}{2}, \\
& =f\left(x_{0}\right)\left|2 t_{1}\right| M_{1}\left(x_{1}, 2-2 t_{2}, x_{2}\right) \quad \text { if } 0 \leqq t_{1} \leqq \frac{1}{2} \text { and } \frac{1}{2} \leqq t_{2} \leqq 1, \\
& =M_{2}\left(x_{0}, 2-2 t_{1}, x_{1}, 2-2 t_{2}, x_{2}\right) \quad \text { if } \frac{1}{2} \leqq t_{1} \leqq 1 \text { and } \\
& \\
B_{2}(M)\left[x_{0}\left|t_{1}\right| x_{1} \mid t_{2}\right]= & {\left[f\left(x_{0}\right)\left|2 t_{1}\right| f\left(x_{1}\right) \mid 2 t_{2}\right] \quad \text { if } 0 \leqq t_{1} \leqq \frac{1}{2} \text { and } 0 \leqq t_{2} \leqq \frac{1}{2}, } \\
& =\left[M_{1}\left(x_{0}, 2-2 t_{1}, x_{1}\right) \mid 2 t_{2}\right] \quad \text { if } \frac{1}{2} \leqq t_{1} \leqq 1 \text { and } 0 \leqq t_{2} \leqq \frac{1}{2}, \\
& =\left[f\left(x_{0}\right) \mid 2 t_{1}\right] \text { if } 0 \leqq t_{1} \leqq \frac{1}{2} \text { and } \frac{1}{2} \leqq t_{2} \leqq 1, \\
& =* \text { if } \frac{1}{2} \leqq t_{1} \leqq 1 \text { and } \frac{1}{2} \leqq t_{2} \leqq 1 .
\end{aligned}
$$

These two examples show clearly why the conditions on the $M_{i}$ in the definition of an $n$-HM map were chosen as they were. (At least for $n=2$.)

Notice that only $M_{0}=f$ was used in defining $B_{1}(M)$ and that $\left\{M_{0}, M_{1}\right\}$ was used in defining $B_{2}(M)$. 
Sugawara then continues this process by induction. His argument actually shows that if $\left\{M_{0}, \ldots, M_{n}\right\}$ is an $n$-HM map from $X$ to $Y$, there are maps

$$
\begin{gathered}
E_{n}(M): E_{n}(X) \rightarrow E_{n}(Y), \\
B_{n+1}(M): B_{n+1}(X) \rightarrow B_{n+1}(Y) .
\end{gathered}
$$

The concept of homotopies between $n$-HM maps is important because of the following theorem:

4.3.4.2 Theorem. Let $\left\{M_{0}, \ldots, M_{n}\right\}$ and $\left\{N_{0}, \ldots, N_{n}\right\}$ be $n-H M$ maps from $X$ to $Y$, where $X$ and $Y$ are $H$-spaces. Suppose $\left\{M_{0}, \ldots, M_{n}\right\}$ and $\left\{N_{0}, \ldots, N_{n}\right\}$ are homotopic (in the sense of 4.3.2). Then

$$
\begin{aligned}
& B_{n+1}(M): B_{n+1}(X) \rightarrow B_{n+1}(Y) \text { and } \\
& B_{n+1}(N): B_{n+1}(X) \rightarrow B_{n+1}(Y) \text { are homotopic. }
\end{aligned}
$$

\subsection{5 n-equivalence of $H$-spaces.}

Definition. Let $X$ and $Y$ be $H$-spaces. Let $\left\{M_{0}, \ldots, M_{n}\right\}$ and $\left\{N_{0}, \ldots, N_{n}\right\}$ be $n$-HM maps from $X$ to $Y$ and from $Y$ to $X$ respectively. Associated with the identity maps $I_{X}, I_{Y}$ of $X$ and $Y$ are $n$-HM maps $\left\{I_{0}, \ldots, I_{n}\right\},\left\{I_{o}^{\prime}, \ldots, I_{n}^{\prime}\right\}$ from $X$ to $X$ and from $Y$ to $Y$ respectively. (For example, $I_{i}: X \times(I \times X)^{i} \rightarrow$ is given by $I_{i}(\omega)=x_{0}(\omega) \cdot x_{1}(\omega) \cdot \cdots \cdot x(\omega)$.)

Suppose $\left\{M_{0}, \ldots, M_{n}\right\}\left\{N_{0}, \ldots, N_{n}\right\}$ and $\left\{N_{0}, \ldots, N_{n}\right\}\left\{M_{0}, \ldots, M_{n}\right\}$ are (n-HM) homotopic to $\left\{I_{o}^{\prime}, \ldots, I_{n}^{\prime}\right\}$ and $\left\{I_{0}, \ldots, I_{n}\right\}$ respectively. Then $X$ and $Y$ will be called n-equivalent.

Later, (8.3), we shall show that if $X$ and $Y$ are CW $H$-spaces, then $X$ and $Y$ are $n$-equivalent if and only if $B_{n+1}(X)$ and $B_{n+1}(Y)$ are homotopy equivalent.

We now proceed to the essential step in the classification theorem:

5. The SHM map $\Omega X \rightarrow F$ for a principal quasifibering $F \rightarrow E \rightarrow X$. We shall construct a SHM map $\left\{M_{0}, \ldots, M_{n}, \ldots\right\}$ from $\Omega X$ to $F$ using certain maps $\left\{H_{0}, H_{1}, \ldots, H_{n}, \ldots\right\}$; the maps $\left\{H_{0}, H_{1}, \ldots, H_{n}, \ldots\right\}$ will be emphasized since they play an important role in the classification theorem. Throughout $\S 5, F \rightarrow E \rightarrow$ $X$ is a fixed principal quasifibering with $X$ a countable CW-complex.

We first construct $H_{0}$.

5.1 THEOREM. There is a mapping $H_{0}: \Omega X \times R^{+} \rightarrow E$ such that $H_{0}(\lambda,|\lambda|) \in F$ and $p H_{0}(\lambda, s) \simeq \lambda(s)$ for $0 \leqq s \leqq|\lambda| .(|\lambda|=$ length of $\lambda$. $)$

Proof. Let

$K=\Omega X=$ the associative Moore loop space,

$\eta_{0}: K \times I \rightarrow X$ given by $\eta_{0}(\lambda, s)=\lambda(|\lambda| \cdot s)$,

$M=K \times \partial I$,

$M^{\prime}=(K \times 0) \cap M$, and 
$\xi_{0}^{\prime}: K \times(0) \rightarrow F \subset E$ given by $\xi_{0}^{\prime}(\lambda, 0)=e \in F$.

Thus we have the commutative diagram

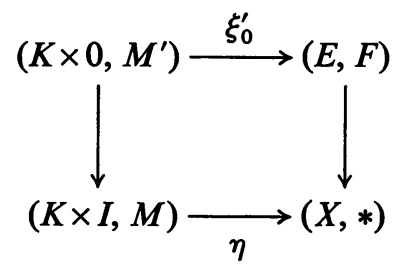

Then by the WACHEP, $\xi_{0}^{\prime}$ can be extended to $\xi_{0}$ and the following diagram is homotopy commutative:



that is, there is a homotopy $Y_{0}:(K \times I \times I, M \times I) \rightarrow(X, *)$ where $Y_{0}(, 0)=p \xi_{0}$ and $Y_{0}(, 1)=\eta$.

Now define $H_{0}: \Omega X \times R^{+} \rightarrow E$ by

$$
\begin{aligned}
H_{0}(\lambda, s) & =\xi_{0}(\lambda, s /|\lambda|) \text { if }|\lambda| \neq 0 \text { and } 0 \leqq s \leqq|\lambda|, \\
& =\xi_{0}(\lambda, 1) \text { if }|\lambda| \neq 0 \text { and } s \geqq|\lambda|, \\
& =e \text { if }|\lambda|=0 .
\end{aligned}
$$

Then $p H_{0}(\lambda, s) \simeq \lambda(s)$ for $0 \leqq s \leqq|\lambda|$, the homotopy being given by

$$
\begin{aligned}
W_{0}(\lambda, s, u) & =Y_{0}(\lambda, s /|\lambda|, u) \text { if }|\lambda| \neq 0 \text { and } 0 \leqq s \leqq|\lambda|, \\
& =* \text { if }|\lambda| \neq 0 \text { and } s \geqq|\lambda|, \text { or if } s=0 .
\end{aligned}
$$

Thus $H_{0}$ satisfies $p H_{0}(\lambda, 0)=e \in F$ and $p H_{0}(\lambda,|\lambda|) \in F$.

The next lemma is the inductive step in constructing $\left\{M_{0}, \ldots, M_{i}, \ldots\right\}$ and $\left\{H_{0}, \ldots, H_{i}, \ldots\right\}$.

5.2 LEMMA. Suppose $n$ is a positive integer, $n \geqq 2$, and for each nonnegative integer $k<n$, there are maps $H_{k}:(\Omega X)_{k} \times R^{+} \rightarrow E, M_{k}:(\Omega X)_{k} \rightarrow F$, and homotopies $W_{k}:(\Omega X)_{k} \times R^{+} \times I \rightarrow X$ such that

$$
\begin{aligned}
& M_{k}(\omega)=M_{k-1}\left(\delta_{i}(\omega)\right) \quad \text { if } t_{i}(\omega)=0, \\
& \quad=M_{i-1}(\omega(0, i-1)) \cdot M_{k-i}(\omega(i, k)) \quad \text { if } t_{i}(\omega)=1 ; \\
& H_{k}(\omega, s)=e \quad \text { if } s=0, \\
& =M_{k}(\omega) \text { if } s \geqq|\omega|, \\
& =H_{k-1}\left(\delta_{i}(\omega), s\right) \text { if } t_{i}(\omega)=0, \\
& =H_{k-i}(\omega(i, k), s) \text { if } t_{i}(\omega)=1 \text { and } 0 \leqq s \leqq|\omega(i, k)|, \\
& =H_{i-1}(\omega(0, i-1), s-|\omega(i, k)|) \cdot M_{k-i}(\omega(i, k)) \quad \text { if } t_{i}(\omega)=1 \text { and } \\
& \quad|\omega(i, k)| \leqq s \leqq|\omega| ;
\end{aligned}
$$




$$
\begin{aligned}
& W_{k}(\omega, s, u)=p H_{k}(\omega, s) \quad \text { if } u=0, \\
& =\lambda_{0}(\omega) \cdots \lambda_{n}(\omega)(s) \quad \text { if } u=1 \text { and } 0 \leqq s \leqq|\omega|, \\
& =* \quad \text { if } s \geqq|\omega| ;
\end{aligned}
$$

Then the statement holds for $n+1$.

In the above, if $\omega=\left(\lambda_{0}, t_{1}, \ldots, t_{n}, \lambda_{n}\right) \in(\Omega X)_{n}=\Omega X \times(I \times \Omega X)^{n}$, then

$\delta_{i}(\omega)=\left(\lambda_{0}, t_{1}, \ldots, t_{i-1}, \lambda_{i-1}, t_{i+1}, \ldots, t_{k}, \lambda_{k}\right)$,

$\omega(\alpha, \beta)=\left(\lambda_{\alpha}, t_{\alpha+1}, \ldots, t_{\beta}, \lambda_{\beta}\right)$,

$|\omega|=\sum_{i=0}^{k}\left|\lambda_{i}(\omega)\right|=$ the sum of lengths of the loops appearing in $\omega$.

Proof. Let

$K=(\Omega X)_{n}=\Omega X *(I \times \Omega X)^{n}$,

$L=K \cap\left\{\omega: t_{i}(\omega) \in \partial I\right.$ for some $\left.i\right\}$,

$M=K \cap \partial I$,

$M^{\prime}=M \cap(K \times(0) \cup L \times I)$.

We will get $H_{n+1}$ by using the WACHEP. We know what $H_{n+1}$ should be when $t_{i}(\omega) \in \partial I$. To get $H_{n+1}$ for all values, we define a normalized version of $H_{n+1}$ restricted to these values, as follows. Define $\xi_{n}^{\prime}: K \times(0) \cup L \times I \rightarrow E$ as follows:

$$
\begin{aligned}
\xi_{n}^{\prime}(\omega, s) & =e \text { if } s=0, \\
& =H_{n-1}\left(\delta_{i}(\omega), s \cdot|\omega|\right) \quad \text { if } t_{i}(\omega)=0, \\
& =H_{n-i}(\omega(i, n), s \cdot|\omega|) \quad \text { if } t_{i}(\omega)=1 \text { and } 0 \leqq s \cdot|\omega| \leqq|\omega(i, n)|, \\
& =H_{i-1}(\omega(0, i-1), s \cdot|\omega|-|\omega(i, n)|) \cdot M_{n-i}(\omega(i, n)) \quad \text { if } t_{i}(\omega)=1 \\
& \quad \text { and }|\omega(i, n)| \leqq s \cdot|\omega| \leqq|\omega| .
\end{aligned}
$$

Equations (2) guarantee that $\xi_{n}^{\prime}$ is well defined.

Construct a homotopy $y_{n}: p \xi_{n}^{\prime}-\eta \mid K \times(0) \cup L \times I$ as follows:

$$
\begin{aligned}
y_{n}(\omega, s, u) & =W_{n-1}\left(\delta_{i}(\omega), s \cdot|\omega|, u\right) \quad \text { if } t_{i}(\omega)=0, \\
& =W_{n-i}(\omega(i, n), s, u) \text { if } t_{i}(\omega)=1 \text { and } 0 \leqq s \leqq|\omega(i, k)|, \\
& =W_{i-1}(\omega(0, i-1), s \cdot|\omega(i, k)|, u) \quad \text { if } t_{i}(\omega)=1 \\
& \text { and }|\omega(i, k)| \leqq s \leqq|\omega| .
\end{aligned}
$$

(Equations (4) guarantee that $y_{n}$ is well defined.) Then $y_{n}$ gives a homotopy between $p \xi_{n}^{\prime}$ and $\eta \mid K \times 0 \cup L \times I$. That is,

$$
\begin{aligned}
& y_{n}:\left((K \times(0) \cup L \times I) \times I, M^{\prime} \times I\right) \rightarrow(X, *), \\
& y_{n}(, 0)=\eta, \\
& y_{n}(, 1)=p \xi_{n}^{\prime} .
\end{aligned}
$$


Hence by the WACHEP, $\xi_{n}^{\prime}$ extends to $\xi_{n}$ and the following diagram is homotopy commutative



That is, there is a homotopy

$$
\begin{gathered}
Y_{n}:(K \times I \times I, M \times I) \rightarrow(X, *), \\
Y_{n}(, 0)=p \xi_{n} \text { and } Y_{n}(, 1)=\eta, \\
Y_{n}(\omega, s, z)=y_{n}(\omega, s, 2 z) \text { if }(\omega, s) \in K \times(0) \cup L \times I \text { and } 0 \leqq z \leqq \frac{1}{2}, \\
=\eta(\omega, s) \text { if }(\omega, s) \in K \times(0) \cup L \times I \text { and } \frac{1}{2} \leqq z \leqq 1
\end{gathered}
$$

Now returning to variable lengths, define $H_{n}:(\Omega X)_{n} \times R^{+} \rightarrow E$ and $W_{n}$ : $(\Omega X)_{n} \times R^{+} \times I \rightarrow X$ by

$$
\begin{aligned}
H_{n}(\omega, s) & =\xi_{n}(\omega, 1) \text { if }|\omega| \neq 0 \text { and } 0 \leqq s \leqq|\omega|, \\
& =\xi_{n}(\omega, 1) \text { if }|\omega| \neq 0 \text { and } s \geqq|\omega|, \\
& =e \text { if }|\omega|=0 \text { and } s \in R^{+} ; \\
W_{n}(\omega, s, u) & =Y_{n}(\omega, s /|\omega|, u) \text { if }|\omega| \neq 0 \text { and } 0 \leqq s \leqq|\omega|, \\
& =* \text { if }|\omega|=0 \text { and } s \in R^{+} \text {or if } 0 \neq|\omega| \leqq s .
\end{aligned}
$$

This completes the inductive definition of $H_{n}, W_{n}$, and $M_{n}$. By the way they were constructed, $H_{k}$ and $W_{k}$ automatically satisfy equations (1)-(3). To check equation (4), if $\omega=\left(\lambda_{0}, t_{1}, \ldots, t_{n}, \lambda_{n}, s\right) \in K \times(0) \cup L \times I$, then (for $|\omega| \neq 0$ )

$$
\begin{aligned}
W_{n}(\omega, s, u)=Y_{n}(\omega, s /|\omega|, u) & =y_{n}(\omega, s /|\omega|, 2 u) \text { if } 0 \leqq u \leqq \frac{1}{2}, \\
& =\eta(\omega, s /|\omega|) \text { if } \frac{1}{2} \leqq u \leqq 1 .
\end{aligned}
$$

Now if $0 \leqq u \leqq \frac{1}{2}$, then equation (6) tells us that (4) is satisfied for $k=n$. If $\frac{1}{2} \leqq u \leqq 1$, then

$$
W_{n}(\omega, s, u)=\eta(\omega, s /|\omega|)=\left(\lambda_{0}(\omega) \cdots \lambda_{n}(\omega)\right)(s)
$$

and so (4) is satisfied in this case too.

Then using this construction, we have

5.3 THEOREM. Given the principal quasifibering with $X$ a countable $\mathrm{CW}$-complex $F \rightarrow E \rightarrow X$ there is a SHM map $\left\{M_{0}, M_{1}, \ldots, M_{n}, \ldots\right\}$ from $\Omega X$ to $F$.

Proof. Construct $\left\{H_{0}, H_{1}, \ldots\right\}$ as in 5.2 and then define for each $n, M_{n}(\omega)=$ $H_{n}(\omega,|\omega|)$. 
6. Classification of principal $F$-bundles. In this section we will show how the maps $\left\{M_{0}, M_{1}, \ldots, M_{i}, \ldots\right\}$ and $\left\{H_{0}, H_{1}, \ldots, H_{i}, \ldots\right\}$ used in constructing a SHM map from $\Omega X$ to $F$ for a principal $F$-bundle $F \rightarrow E \rightarrow X$ can be used to classify the fibering.

Before carrying this out, it should be pointed out that Stasheff [12] has proved a classification theorem for fiber spaces $F \rightarrow E \rightarrow X$ ( $F$ is the fiber over the base point of $X$, not an $H$-space) in terms of $A_{\infty}$-actions of $\Omega B$ on $F$. The fiber spaces are classified up to fiber homotopy equivalence. Some of the techniques in this classification theorem are the same as Stasheff's. Stasheff defines, for an associative $H$-space $Y$, an $A_{\infty}$-action of $Y$ on a space $F$ to be a collection of maps

such that

$$
\left\{\theta_{i}:(Y \times I)^{i-1} \times Y \times F \rightarrow F\right\}
$$

$$
\begin{aligned}
\theta_{i}\left(y_{0}, t_{1},\right. & \left.y_{1}, \ldots, t_{i-1}, y_{i-1}, f\right) \\
& =\theta_{i-1}\left(y_{0}, \ldots, t_{j-1}, y_{j-1} y_{j}, t_{j+1}, \ldots, t_{i-1}, y_{i-1}, f\right) \text { if } t_{j}=1, \\
& =\theta_{j}\left(y_{0}, \ldots, t_{j-1}, y_{j-1}, \theta_{i-j}\left(y_{j}, t_{j+1}, \ldots, t_{i-1}, y_{i-1}, f\right)\right) \text { if } t_{j}=0 .
\end{aligned}
$$

He then defines, for a fiber space $p: E \rightarrow X$, a transport to be an $A_{\infty}$-action $\left\{\theta_{i}\right\}$ of $\Omega B$ on $F$ (the fiber over the base point of $X$ ) such that for each $\lambda \in \Omega B$, $\theta_{i}(\lambda, \quad): F \rightarrow F$ is a homotopy equivalence. Stasheff proves

THEOREM A. Given a fiber space $E \rightarrow X$, there is a transport (constructed in a nontrivial way). Given a transport, there is a corresponding fiber space $\left(E_{\theta} \rightarrow X\right)$.

Given a fibre space $F \rightarrow E \rightarrow X$, there is an associated principal fiber space $H(F) \rightarrow \operatorname{Prin} E \rightarrow X$ where $\widetilde{E}=\operatorname{Prin} E=\left\{\varphi: F \rightarrow p^{-1}(x) \mid \varphi\right.$ is a homotopy equivalence $\}$ and $H(F)=\{\varphi: F \rightarrow F \mid \varphi$ is a homotopy equivalence $\}$. We construct a SHM map $\left\{M_{0}, \ldots, M_{i}, \ldots\right\}$ from $\Omega X$ to $H(F)$. We shall see that this classifies Prin $E \rightarrow$ $X$. Stasheff could obtain his transport by defining

$$
\Theta_{i}\left(\lambda_{0}, t_{1}, \lambda_{1}, \ldots, t_{i-1}, \lambda_{i-1}, f\right)=M_{i}\left(\lambda_{0}, 1-t_{1}, \ldots, 1-t_{i-1}, \lambda_{i-1}\right)(f) ;
$$

this would classify $E \rightarrow X$. In fact, this point of view can be used to show that there is a one-to-one correspondence between the set of equivalence classes of fiber spaces and their associated principal fibrations. An alternate approach appears in $\S 7$.

6.1 The induced fibering. Given a classifying map $X \rightarrow B_{F}$ one does not directly take an "induced" fibering. In general one does not take an induced quasifibration and it is not known if one could in this special case. So instead one considers $X \rightarrow B_{F}$ as a map $B_{\Omega X} \rightarrow B_{F}$ which corresponds to a SHM map $\Omega X \rightarrow F$.

In $\S 5$, we showed how to construct the SHM map $\left\{M_{0}, M_{1}, \ldots, M_{i}, \ldots\right\}$ from $\Omega X$ to $F$ for a given principal $F$-quasifibering $F \rightarrow E \rightarrow X$. We will now show how to construct a principal $F$-bundle $F \rightarrow E_{M} \rightarrow X$ when given a SHM map $\left\{M_{0}, M_{1}, \ldots, M_{i}, \ldots\right\}$ from $\Omega X$ to $F$. 
The motivation for this definition comes from the construction of the SHM map $\left\{M_{0}, M_{1}, \ldots, M_{n}, \ldots\right\}$ from $\Omega X$ to $F$ in $\S 5$. That is these maps $\left\{M_{0}, M_{1}, \ldots, M_{n}, \ldots\right\}$ were defined in terms of maps $\left\{H_{0}, H_{1}, \ldots, H_{n}, \ldots\right\}$ which satisfied equations (1)-(4). Now given a SHM map $\left\{M_{0}, M_{1}, \ldots, M_{n}, \ldots\right\}$ from $\Omega X$ to $F$ we will form a space $E_{M}$ so that $E_{M}$ is the union of an increasing sequence $E_{0} \subset E_{1} \subset \ldots \subset E_{n} \subset \ldots$ where each $E_{n}$ is an identification space $(\Omega X)_{n} \times R^{+} \times F / \sim$ so that if $q(\omega, s, f)$ is the class in $E_{n}$, the $q_{n}$ 's satisfy the same equations as do the $H_{n}$ 's.

6.2 The construction of $F \rightarrow E_{M} \rightarrow X$. Suppose $X$ is a countable CW-space (with base point $*$ ) and $F$ is an (associative) countable $\mathrm{CW} H$-space and that $\left\{M_{0}, M_{1}, \ldots, M_{i}, \ldots\right\}$ is a SHM map from $\Omega X$ to $F$. Define $E_{0}=F$ and $p_{0}: E_{0} \rightarrow X$ to be the projection of $F$ onto the base point of $X$. Define $E_{1}$ to be the identification space from $\Omega X \times R^{+} \times F$ by identifying

$\left(\lambda_{0}, s, f\right)$ with $f$ if $s=0$,

$\left(\lambda_{0}, s, f\right)$ with $f \cdot M_{0}\left(\lambda_{0}\right)$ if $s \geqq\left|\lambda_{0}\right|$.

We will write the equivalence class represented by $\left(\lambda_{0}, s, f\right)$ as $q\left(\lambda_{0}, s, f\right)$. Define $p_{1}: E_{1} \rightarrow X$ by

$$
\begin{aligned}
p\left(q\left(\lambda_{0}, s, f\right)\right) & =\lambda_{0}(s) \quad \text { if } 0 \leqq s \leqq\left|\lambda_{0}\right|, \\
& =* \text { if }\left|\lambda_{0}\right| \leqq s .
\end{aligned}
$$

In general, for $n \geqq 1, E_{n+1}$ is the identification space formed from

$$
(\Omega X)_{n} \times R^{+} \times F \stackrel{q}{\longrightarrow} E_{n+1}
$$

with the following identifications:

$$
\begin{aligned}
q(\omega, s, f) & =f \in F \quad \text { if } s=0 \\
& =q\left(\delta_{i}(\omega), s, f\right) \in E_{n}(X) \quad \text { if } t_{i}(\omega)=0 \\
& =q\left(\omega(0, i-1), s-|\omega(i, n)|, f \cdot M_{n-i}(\omega(i, n))\right) \in F_{i-2}(X) \\
& =q(\omega(i, n), s, f) \in E_{n-1+i}(X) \quad \text { if } t_{i}(\omega)=1 \text { and } 0 \leqq s \leqq|\omega(i, n)| \\
& =f \cdot M_{n}(\omega) \in F \quad \text { if } s \geqq|\omega| .
\end{aligned}
$$

Define $p_{n+1}: E_{n+1}(X) \rightarrow X$ by

$$
\begin{aligned}
p_{n+1}(q(\omega, s, f)) & =\lambda_{0}(\omega) \cdots \lambda_{n}(\omega)(s) \quad \text { if } 0 \leqq s \leqq|\omega|, \\
& =* \quad \text { if } s \geqq|\omega| .
\end{aligned}
$$

We may consider $E_{0} \subset E_{1} \subset \ldots \subset E_{n} \subset \ldots$. Let $E_{M}$ be the union of the $E_{n}$ (with the limit topology) and let $p: E_{M} \rightarrow X$ be the union of the $p_{n}$.

6.3 TheORem. $F \rightarrow E_{M} \rightarrow X$ is an F-bundle.

We show "local triviality". For $x \in X$, choose an open cell $U(x)$ in $S$. Let $(\omega, s, f) \in$ $(\Omega X)_{n} \times R^{+} \times F$ so that $p q(\omega, s, f)=x$. Thus $\lambda_{0}(\omega) \cdots \lambda_{n}(\omega)(s)=x$. 
Now let $y \in U(x)$. Let $d(x, y)$ be the distance from $x$ to $y$ in the cell $U(x)$. Let $\eta_{y}$ be the path in $U(x)$ starting at $x$ and ending at $y$ in time $d(x, y)$, along the line from $x$ to $y$ in $U(x)$.

What we do is replace the loop in which $x$ lies by a loop which takes a deviation to $y$, then back to $x$ again, and then continues along itself as before. More precisely, if $0 \leqq s \leqq\left|\lambda_{n}(\omega)\right|$, let $j=n$. If $s>|\omega|$, let $j=-1$. Otherwise, choose $j$ so that $|\omega(j+1, n)| \leqq s \leqq|\omega(j, n)|$.

Case 1. $j \neq-1$. Define a loop ${ }_{y} \lambda_{j}$ with length $\left|\lambda_{j}\right|+2 d(x, y)$ by

$$
\begin{aligned}
{ }_{y} \lambda_{j}(t) & =\lambda_{j}(t) \quad \text { if } 0 \leqq t \leqq u \\
& =\lambda_{y}(t-u) \quad \text { if } u \leqq t \leqq u+d(x, y) \\
& =\lambda_{y}(u+2 d(x, y)-t) \quad \text { if } u+d(x, y) \leqq t \leqq u+2 d(x, y) \\
& =\lambda_{j}(t-2 d(x, y)) \quad \text { if } u+2 d(x, y) \leqq t \leqq\left|\lambda_{j}\right|+2 d(x, y)
\end{aligned}
$$

where $u=s-\omega(j+1, n)$.

Case 2. $j=-1$. Let ${ }_{y} \lambda_{-1}$ be the loop defined by

$$
\begin{aligned}
{ }_{y} \lambda_{-1}(t) & =\lambda_{y}(t), \quad 0 \leqq t \leqq d(x, y), \\
& =\lambda_{y}(2 d(x, y)-t), \quad d(x, y) \leqq t \leqq 2 d(x, y) .
\end{aligned}
$$

Now define $s: U(x) \rightarrow E_{M}$ by

$$
\begin{aligned}
s(y) & =q\left(\omega^{\prime}, s+d(x, y), f\right) \quad \text { if } j \neq-1, \\
& =q\left({ }_{y} \lambda_{-1}, s-|\omega|, M_{n}(\omega) \cdot f\right) \quad \text { if } j=-1,
\end{aligned}
$$

where $\omega^{\prime}$ represents $\omega=\left(\lambda_{0}(\omega), t_{1}, \ldots, t_{n}, \lambda_{n}(\omega)\right)$ with $\lambda_{j}$ replaced by ${ }_{y} \lambda_{j}$.

Then $s$ gives the desired local cross section, and we have the map $\varphi_{U}: p^{-1}(U) \rightarrow$ $U \times F$ given by

$$
q(\omega, s, f) \rightarrow\left(\lambda_{0}(\omega) \cdots \lambda_{n}(\omega)(s), M_{n}\left(\lambda_{0}(\omega), t_{1}, \ldots, t_{n}, \lambda_{n}(\omega)\right) \cdot f\right) .
$$

One checks directly that the homotopies involved are principal.

We now state one form of the main theorem of this section:

6.4 THEOREM. Let $X$ be a countable CW-space and $F$ a countable connected $\mathrm{CW} H$-space. The equivalence classes of principal $F$-bundles over $X$ are in one-to-one correspondence with (SHM) homotopy classes of SHM maps from $\Omega X$ to $F$. The correspondence is given by constructing the $S H M$ map $\left\{M_{0}, M_{1}, \ldots, M_{i}, \ldots\right\}$ from $\Omega X$ to $F$ when given a principal $F$-bundle $F \rightarrow E \rightarrow X$ as in $\S 5$.

The proof will follow the next several theorems. To show the correspondence is well defined, we prove

6.5 Theorem. Suppose $F \rightarrow E^{1} \rightarrow X$ and $F \rightarrow E^{2} \rightarrow X$ are equivalent principal F-bundles. Let $M^{1}=\left\{M_{0}^{1}, M_{1}^{1}, \ldots, M_{i}^{1}, \ldots\right\}$ and $M^{2}=\left\{M_{0}^{2}, M_{1}^{2}, \ldots, M_{i}^{2}, \ldots\right\}$ be 
the SHM maps from $\Omega X$ to $F$ constructed for the two fiberings as in $\S 5$. Then $M^{1}$ and $M^{2}$ are $(S H M)$ homotopic.

Proof. Recall that in the construction of $M^{1}$ and $M^{2}(\S 5)$ we had, for $k=$ $0,1,2, \ldots$, maps $H_{k}^{i}:(\Omega B)_{k} \times R^{+} \rightarrow E^{i}(i=1,2)$ and homotopies in the base space

$$
W_{k}^{i}:(\Omega B)_{k} \times R^{+} \times I \rightarrow X \quad(i=1,2)
$$

so that

$$
\begin{aligned}
H_{k}^{i}(\omega, s)= & H_{j-1}^{i}(\omega(0, j-1), s-|\omega(j, k)|) \cdot M_{k-j}(\omega(j, k)) \quad \text { if } t_{j}(\omega)=1 \text { and } \\
& \quad(|\omega(j, k)|) \leqq s \leqq|(\omega(0, k))|, \\
= & H_{k-j}^{i}(\omega(j, k), s) \text { if } t_{j}(\omega)=1 \text { and } 0 \leqq s \leqq|\omega(j, k)|, \\
= & H_{k-1}^{i}\left(\delta_{j}(\omega), s\right) \text { if } t_{j}(\omega)=0, \\
= & H_{k}^{i}(\omega,|\omega|) \text { if } s \geqq|\omega|,
\end{aligned}
$$

and $W_{k}^{i}$ is a homotopy between $p H_{k}^{i}(\omega, s)$ and $\lambda_{0}(\omega) \cdots \lambda_{k}(\omega)(s)$. That is,

$$
\begin{aligned}
W_{k}^{i}(\omega, s, u) & =p H_{k}^{i}(\omega, s) \text { if } u=1, \\
& =\lambda_{0}(\omega) \cdots \lambda_{k}(\omega)(s) \text { if } u=0 \text { and } 0 \leqq s \leqq|\omega|, \\
& =* \quad \text { if } s \geqq|\omega| .
\end{aligned}
$$

Since $F \rightarrow E^{1} \rightarrow X$ and $F \rightarrow E^{2} \rightarrow X$ are equivalent, there are principal maps $g^{1}: E^{1} \rightarrow E^{2}$ and $g^{2}: E^{2} \rightarrow E^{1}$ such that $g^{2} g^{1} \simeq$ id and $g^{1} g^{2} \simeq$ id, both homotopies being given by principal homotopies. We now construct maps $\left\{\psi_{i}\right\}$ similar to the way

$$
\left\{H_{0}^{i}, H_{1}^{i}, \ldots, H_{n}^{i}, \ldots\right\}
$$

were constructed: Define $\psi_{0}^{\prime}: I \times \Omega X \times(0) \cup \partial I \times \Omega X \times R^{+} \rightarrow E^{2}$ by

$$
\begin{aligned}
\psi_{0}^{\prime}\left(t_{0}, \lambda_{0}, s\right) & =e \text { if } s=0\left(e \in F \subset E^{2} \text { is the base point of } F\right) \\
& =H_{0}^{2}\left(\lambda_{0}, s\right) \text { if } t_{0}=0 \\
& =g^{1} H_{0}^{1}\left(\lambda_{0}, s\right) \text { if } t_{0}=1
\end{aligned}
$$

Also define $G_{0}: I \times \Omega X \times R^{+} \rightarrow X$ by

$$
\begin{aligned}
& G_{0}\left(t_{0}, \lambda_{0}, s\right)=W_{0}^{1}\left(\lambda_{0}, s, 2\left(t_{0}-\frac{1}{2}\right)\right) \quad \text { if } \frac{1}{2} \leqq t_{0} \leqq 1, \\
& =W_{0}^{2}\left(\lambda_{0}, s, 2\left(\frac{1}{2}-t_{0}\right)\right) \text { if } 0 \leqq t_{0} \leqq \frac{1}{2} \text {. }
\end{aligned}
$$

By normalizing $G_{0}$, we get $\eta_{0}: I \times \Omega X \times I \rightarrow X$ defined by

$$
\eta_{0}\left(t_{0}, \lambda_{0}, s\right)=G_{0}\left(t_{0}, \lambda_{0}, s \cdot|\lambda|\right) \text {. }
$$

Also, by normalizing, we get ${ }_{n} \psi_{0}^{\prime}: I \times \Omega X \times(0) \cup \partial I \times \Omega X \times I \rightarrow E^{2}$ defined by

$$
{ }_{n} \psi_{0}^{\prime}\left(t_{0}, \lambda_{0}, s\right)=\psi_{0}^{\prime}\left(t_{0}, \lambda_{0}, s \cdot\left|\lambda_{0}\right|\right) .
$$

By applying the WACHEP, as before, we get an extension

$$
{ }_{n} \psi_{0}:(I \times \Omega X \times I, I \times \Omega X \times \partial I) \rightarrow\left(E_{2}, F\right)
$$


and a homotopy between $p_{2}{ }_{n} \psi_{0}$ and $\eta_{0}$, that is

$$
{ }_{n} Z_{t}^{0}:(I \times \Omega X \times I, I \times \Omega X \times \partial I) \rightarrow(X, *) \text { for } 0 \leqq t \leqq 1
$$

such that ${ }_{n} Z_{1}^{0}=p_{2}{ }_{n} \psi_{0}$ and ${ }_{n} Z_{0}^{0}=\eta_{0} .\left(p_{2}: E^{2} \rightarrow X\right.$ is the projection in the fibering $F \rightarrow E^{2} \rightarrow X$.) Now returning to variable lengths, define $\psi_{0}: I \times \Omega X \times R^{+} \rightarrow E^{2}$ by

$$
\begin{aligned}
\psi_{0}\left(t_{0}, \lambda_{0}, s\right) & ={ }_{n} \psi_{0}\left(t_{0}, \lambda_{0}, s /\left|\lambda_{0}\right|\right) \quad \text { if }\left|\lambda_{0}\right| \neq 0 \text { and } 0 \leqq s \leqq\left|\lambda_{0}\right| \\
& =e \text { if }\left|\lambda_{0}\right|=0 \text { and } s \in R^{+} \\
& ={ }_{n} \psi_{0}\left(t_{0}, \lambda_{0}, 1\right) \text { if }\left|\lambda_{0}\right| \neq 0 \text { and } s \geqq\left|\lambda_{0}\right|
\end{aligned}
$$

${ }_{n} Z_{t}^{0}$ yields a mapping $Z_{t}^{0}: I \times \Omega X \times R^{+} \rightarrow X$ in a similar way.

$h_{0}: I \times \Omega X \rightarrow F$ is defined by $h_{0}\left(t_{0}, \lambda_{0}\right)=\psi_{0}\left(t_{0}, \lambda_{0},\left|\lambda_{0}\right|\right) . h_{0}$ is a homotopy between $M_{0}^{2}\left(\lambda_{0}\right)$ and $M_{0}^{1}\left(\lambda_{0}\right)$.

By induction, and arguing almost identically to the proof of 5.3, it follows that for each $n$ there is a mapping $\psi_{n}: I \times(\Omega X)_{n} \times R^{+} \rightarrow E^{2}$ such that $\psi_{n}\left(t_{0}, \omega,|\omega|\right) \in F$ and

$$
\begin{aligned}
\psi_{n}\left(t_{0}, \omega, s\right) & =e \quad \text { if } s=0, \\
& =\psi_{n-1}\left(t_{0}, \delta_{i}(\omega), s\right) \quad \text { if } t_{\imath}(\omega)=0 \\
& =\psi_{i-1}\left(t_{0}, \omega(0, i-1), s-|\omega(i, n)|\right) \cdot \psi_{n-i}\left(t_{0}, \omega(i, n),|\omega(i, n)|\right) \\
& \quad \text { if } t_{i}(\omega)=1 \text { and }|\omega(i, n)| \leqq s \leqq|\omega(0, n)|, \\
& =\psi_{n-i}\left(t_{0}, \omega(i, n), s\right) \quad \text { if } t_{i}=1 \text { and } 0 \leqq s \leqq|\omega(i, n)|, \\
& =H_{n}^{2}(\omega, s) \quad \text { if } t_{0}=0 \\
& =g^{1} H_{n}^{1}(\omega, s) \quad \text { if } t_{0}=1, \\
& =\psi_{n}\left(t_{0}, \omega,|\omega|\right) \quad \text { if } s \geqq|\omega| .
\end{aligned}
$$

It also follows that for $n$ there is a homotopy $Z_{t}^{n}: I \times(\Omega X)_{n} \times R^{+} \rightarrow X$ such that

$$
\begin{aligned}
Z_{t}^{n}\left(t_{0}, \omega, s\right) & =p_{2} \psi_{n}\left(t_{0}, \omega, s\right) \quad \text { if } t=1, \\
& =\lambda_{0}(\omega) \cdots \lambda_{n}(\omega)(s) \quad \text { if } t=0
\end{aligned}
$$

$h_{n}$ is obtained by setting $h_{n}\left(t_{0}, \omega\right)=\psi_{n}\left(t_{0}, \omega,|\omega|\right)$.

Then $h=\left\{h_{0}, h_{1}, \ldots, h_{n}, \ldots\right\}$ is a (SHM) homotopy between

$$
M^{1}=\left\{M_{0}^{1}, M_{1}^{1}, \ldots, M_{n}^{1}, \ldots\right\} \text { and } M^{2}=\left\{M_{0}^{2}, M_{1}^{2}, \ldots, M_{n}^{2}, \ldots\right\} .
$$

Having shown that correspondence from equivalence classes of $F$-bundles over $X$ to (SHM) homotopy classes of SHM maps from $\Omega X$ to $F$ is well defined, we show that the correspondence is onto:

6.6 Theorem. Suppose $X$ is a $\mathrm{CW}$-space and $F$ is an associative $\mathrm{CW} H$-space. Suppose $M=\left\{M_{0}, M_{1}, \ldots, M_{i}, \ldots\right\}$ is a $S H M$ map from $\Omega X$ to $F$. Let $F \rightarrow E_{M} \rightarrow X$ be the principal $F$-bundle induced by $M(6.2)$. Let $M^{\prime}=\left\{M_{0}^{\prime}, M_{1}^{\prime}, \ldots, M_{n}^{\prime}, \ldots\right\}$ be the SHM map from $\Omega X$ to $F$ induced by the principal $F$-bundle $F \rightarrow E_{M} \rightarrow X$ (see 5.3). Then $M=\left\{M_{0}, \ldots, M_{n}, \ldots\right\}$ and $M^{\prime}=\left\{M_{0}^{\prime}, \ldots, M_{n}^{\prime}, \ldots\right\}$ are $(S H M)$ homotopic. 
Proof. For each $n$, define $H_{n}:(\Omega X)_{n} \times R^{+} \rightarrow E_{M}$ by $H_{n}(\omega, s)=q(\omega, s, e)$. (Recall $q(\omega, s, e)$ is the equivalence class containing $(\omega, s, e)$.) Because of the identifications in $E_{M}$, we have the equations

$$
\begin{aligned}
H_{n}(\omega, s) & =e \text { if } s=0, \\
& =M_{n}(\omega) \text { if } s \geqq|\omega|, \\
& =H_{i-1}(\omega(0, i-1), s-|\omega(i, n)|) \cdot M_{n-i}(\omega(i, n)) \quad \text { if } t_{i}(\omega)=1 \text { and } \\
& =H_{n-i}(\omega(i, n), s) \text { if } t_{i}(\omega)=1 \text { and } 0 \leqq s \leqq|(\omega(i, n))|, \\
& =H_{n-1}\left(\delta_{i}(\omega)\right) \text { if } t_{i}(\omega)=0, \\
& =H_{n}(\omega,|\omega|) \text { if } s \geqq|\omega|,
\end{aligned}
$$

and $p H_{n}(\omega, s)=\lambda_{0}(\omega) \cdots \lambda_{n}(\omega)(s)$. By the construction of $M^{\prime}$, in $E_{M}$ we have maps $H_{n}^{\prime}:(\Omega X)_{n} \times R^{+} \rightarrow E_{M}$ satisfying the same equation as above, except with primes on the $H$ 's and $M$ 's. And

$$
p H_{n}^{\prime}(\omega, s) \simeq \lambda_{0}(\omega) \cdots \lambda_{n}(\omega)(s) .
$$

For all $n, \psi_{n}: I \times(\Omega X)_{n} \times R^{+} \rightarrow E_{M}$ are constructed by induction exactly the same way that maps $\psi_{n}: I \times(\Omega X)_{n} \times R^{+} \rightarrow E^{2}$ were constructed in 6.5 , the only difference being that $H_{n}^{2}(\omega, s)$ is replaced by $H_{n}^{\prime}(\omega, s)$ and $g^{1} H_{n}^{1}(\omega, s)$ is replaced by $H_{n}(\omega, s)$. As before, $h_{n}\left(t_{0}, \omega\right)=\psi_{n}\left(t_{0}, \omega,|\omega|\right)$ gives the required homotopy between $M$ and $M^{\prime}$. To complete the proof of the classification theorem (Theorem 6.4) we need to show that the correspondence is one-to-one, that is

6.7 Theorem. Suppose $F \rightarrow E^{1} \rightarrow X$ and $F \rightarrow E^{2} \rightarrow X$ are principal $F$-bundles. Let $M^{1}=\left\{M_{0}^{1}, M_{1}^{1}, \ldots, M_{i}^{1}, \ldots\right\}$ and $M^{2}=\left\{M_{0}^{2}, M_{1}^{2}, \ldots, M_{i}^{2}, \ldots\right\}$ be the $S H M$ maps from $\Omega X$ to $F$ constructed for the two quasifiberings $F \rightarrow E^{1} \rightarrow X$ and $F \rightarrow E^{2} \rightarrow X$ respectively (5.3). Suppose $M^{1}$ and $M^{2}$ are (SHM) homotopic. Then $F \rightarrow E^{1} \rightarrow X$ and $F \rightarrow E^{2} \rightarrow X$ are equivalent.

This theorem follows from the next two theorems.

6.8 TheOREM. Suppose $F \rightarrow E^{1} \rightarrow X$ and $F \rightarrow E^{2} \rightarrow X$ are principal $F$-bundles. Let $M^{1}=\left\{M_{0}^{1}, M_{1}^{1}, \ldots, M_{i}^{1}, \ldots\right\}$ and $M^{2}=\left\{M_{0}^{2}, M_{1}^{2}, \ldots, M_{i}^{2}, \ldots\right\}$ be the SHM maps from $\Omega X$ to $F$ constructed for the two principal $F$-bundles $F \rightarrow E^{1} \rightarrow X$ and $F \rightarrow E^{2} \rightarrow X$ respectively. Suppose $M^{1}$ and $M^{2}$ are (SHM) homotopic. Then the two principal F-bundles $F \rightarrow E_{M^{1}} \rightarrow X$ and $F \rightarrow E_{M^{2}} \rightarrow X$ are equivalent.

6.9 TheOREM. Suppose $F \rightarrow E \rightarrow X$ is a principal F-bundle. Let

$$
M=\left\{M_{0}, M_{1}, \ldots, M_{i}, \ldots\right\}
$$

be the SHM maps from $\Omega X$ to $F$ constructed from $F \rightarrow E \rightarrow X$. Then $F \rightarrow E \rightarrow X$ and $F \rightarrow E_{M} \rightarrow X$ are equivalent principal $F$-bundles.

In the preceding three theorems, $X$ and $F$ are assumed, as usual, to be countable $\mathrm{CW}$-complexes, and $F$ is assumed to be connected. 
Proof of 6.8. Recall that elements of $E_{M^{1}}$ are written in the form $q(\omega, s, f)$ where $\omega \in(\Omega X)_{n}$ for some $n, s \in R^{+}, f \in F$. We will now write these elements as $q^{1}(\omega, s, f)$. Similarly, elements in $E_{M^{2}}$ are written in the form $q^{2}(\omega, s, f)$ where $\omega \in(\Omega X)_{n}$ for some $n, s \in R^{+}$, and $f \in F$. Recall that because of the identifications in $E_{M^{1}}, q(\omega, s, f)=f \cdot M_{n}(\omega)$ if $\omega \in(\Omega X)_{n}, s=|\omega|$, and $f \in F$.

Since $M^{1}$ and $M^{2}$ are (SHM) homotopic, there is a sequence of maps $\left\{h_{0}, h_{1}, \ldots, h_{n}, \ldots\right\}$ where $h_{n}: I \times(\Omega X)_{n} \rightarrow F$ is such that $h_{n}(0, \omega)=M_{n}^{1}(\omega)$, $h_{n}(1, \omega)=M_{n}^{2}(\omega)$, and for each $t_{0} \in I$, the sequence $h_{n}\left(t_{0},\right):(\Omega X)_{n} \rightarrow F$ forms a SHM from $\Omega X$ to $F$.

Recall that $E_{M^{1}}$ is the union of an increasing sequence

$$
F=E_{M^{1}}^{0} \subset E_{M^{1}}^{1} \subset E_{M^{1}}^{2} \subset \ldots \subset E_{M^{1}}^{n} \subset \ldots \subset E_{M^{1}} .
$$

( $E_{M^{1}}^{n}$ is the set of $q^{1}(\omega, s, f)$ such that $\omega \in(\Omega X)_{k}$ where $0 \leqq k \leqq n$.) We will define a map by induction from $E_{M^{1}}$ to $E_{M^{2}}$.

Define $\xi_{1}: I \times \Omega X \times R^{+} \rightarrow E_{M^{2}}$ as follows: First define ${ }_{n} \xi_{1}^{\prime}: I \times(\Omega X) \times(0) \cup$ $(0) \times(\Omega X) \times \partial I \rightarrow E_{M^{2}}$ by

$$
\begin{aligned}
{ }_{n} \xi_{1}^{\prime}\left(t_{0}, \lambda_{0}, s\right) & =e \quad \text { if } s=0, t_{0} \in I, \lambda_{0} \in \Omega X, \\
& =q^{2}\left(\lambda_{0}, s \cdot\left|\lambda_{0}\right|, e\right) \quad \text { if } t_{0}=0, \\
& =h_{0}\left(t_{0}, \lambda_{0}\right) \quad \text { if } s=1 .
\end{aligned}
$$

${ }_{n} \eta: I \times \Omega X \times I \rightarrow X$ is defined by

$$
{ }_{n} \eta\left(t_{0}, \lambda_{0}, s\right)=\lambda_{0}\left(s \cdot\left|\lambda_{0}\right|\right) .
$$

By the now familiar use of the WACHEP, ${ }_{n} \xi_{1}^{\prime}$ is extended to ${ }_{n} \xi_{1}: I \times \Omega X \times I \rightarrow E_{M^{2}}$ and hence $\xi_{1}^{\prime}$ is extended to $\xi_{1}: I \times \Omega X \times R^{+} \rightarrow E_{M^{2}}$. In particular, $\xi_{1}\left(1, \lambda_{0}, 0\right)=e$ and

$$
\xi_{1}\left(1, \lambda_{0},\left|\lambda_{0}\right|\right)=h_{0}\left(1, \lambda_{0}\right)=M_{0}^{2}\left(\lambda_{0}\right) \text {. }
$$

Hence $\xi_{1}$ induces a mapping $g_{1}: E_{M^{1}}^{1} \rightarrow E_{M^{2}}$ by defining

$$
g_{1}\left(q^{1}\left(\lambda_{0}, s, f\right)\right)=\xi_{1}\left(1, \lambda_{0}, s\right) \cdot f .
$$

By induction, arguing as before, (5.1), it follows that for each $n$, there is $\xi_{n}: I \times(\Omega X)_{n-1} \times R^{+} \rightarrow E_{M^{2}}$ so that

$$
\begin{aligned}
\xi_{n}\left(t_{0}, \omega, s\right) & =e \quad \text { if } s=0, \\
& =q^{2}(\omega, s) \quad \text { if } t_{0}=0, \\
& =\xi_{n-1}\left(t_{0}, \delta_{i}(\omega), s\right) \quad \text { if } t_{i}(\omega)=0, \\
& =\xi_{n-i}\left(t_{0}, \omega(i, n-1), s\right) \text { if } t_{i}(\omega)=1 \text { and } 0 \leqq s \leqq|\omega(i, n-1)|, \\
& =\xi_{i-1}(\omega(0, i-1), s-|\omega(i, n-1)|) \cdot \xi_{n-i}(\omega(i, n-1),|\omega(i, n-1)|) \\
& \quad \text { if } t_{i}(\omega)=1 \text { and }|\omega(i, n-1)| \leqq s \leqq|\omega(0, n-1)|, \\
= & h_{n-1}\left(t_{0}, \omega\right) \quad \text { if } s \geqq|\omega(0, n-1)| .
\end{aligned}
$$


The inductive argument is almost identical to that used before. $\xi_{n}^{\prime}$ is defined for values $s=0, s=|\omega|, t_{i}(\omega) \in \partial\left(I^{n-1}\right)$, and $t_{0}=0$ by the above equations. $\xi_{n}^{\prime}$, as a function of $s$, has projection homotopic to $\lambda_{0}(\omega) \cdots \lambda_{n}(\omega)(s)$. Then a map $\eta: I \times(\Omega X)_{n-1} \times R^{+} \rightarrow X$ is defined. By normalizing, using the WACHEP, and returning to variable lengths, $\xi_{n}^{\prime}$ is extended to $\xi_{n}$.

Having thus defined $\left\{\xi_{1}, \xi_{2}, \ldots, \xi_{n}, \ldots\right\}$ the maps $g_{n}: E_{M^{1}}^{n} \rightarrow E_{M^{2}}$ are defined for all $n$, and $g_{n+1}$ is an extension of $g_{n}$ for all $n$. $g_{n}$ is defined by

$$
g_{n}\left(q^{1}(\omega, s, f)\right)=\xi_{n}(1, \omega, s) \cdot f \text { if } \omega \in(\Omega X)_{n-1}, s \in R^{+}, f \in F .
$$

Thus the maps $\left\{g_{1}, \ldots, g_{n}, \ldots\right\}$ induce $g: E_{M^{1}} \rightarrow E_{M^{2}}$ which is a principal map. Similarly there are maps $\left\{\xi_{1}^{\prime}, \ldots, \xi_{n}^{\prime}, \ldots\right\}$ giving $g_{n}^{\prime}: E_{M^{2}}^{n} \rightarrow E_{M^{1}}$ and hence $g^{\prime}: E_{M^{2}} \rightarrow E_{M^{1}}$.

To finish the proof, one can show, using the same type of arguments, that $g g^{\prime}$ and $g^{\prime} g$ are homotopic to the identity mappings, the homotopies being principal homotopies lying over the identity.

Proof of 6.9. Recall that the SHM map $M=\left\{M_{0}, M_{1}, \ldots, M_{i}, \ldots\right\}$ from $\Omega X$ to to $F$ was defined in terms of maps $\left\{H_{0}, H_{1}, \ldots, H_{i}, \ldots\right\}$ where $H_{i}:(\Omega X)_{i} \times R^{+} \rightarrow E$ and also that given the SHM map $M=\left\{M_{0}, M_{1}, \ldots, M_{i}, \ldots\right\}$ from $\Omega X$ to $F$, the identification space $E_{M}$ was formed so that representative elements $q(\omega, s, f)$ satisfy the same kind of equations that the $H_{i}(\omega, s) \cdot f$ satisfy (see 6.2 and 5.1).

Thus we have a principal map $g: E_{M} \rightarrow E$, where

$$
g(q(\omega, s, f))=H_{n}(\omega, s) \cdot f \quad \text { if } \omega \in(\Omega X)_{n}, s \in R^{+}, f \in F .
$$

Since $p H_{n}(\omega, s)=\lambda_{0}(\omega) \cdots \lambda_{n}(\omega)(s)$ if $\omega \in(\Omega X)_{n}, s \in R^{+}$and $p q(\omega, s)=\lambda_{0}(\omega) \cdots$ $\lambda_{n}(\omega)(s)$, we see that $g$ does not lie over the identity mapping of $X$, but lies over a map that is homotopic to the identity mapping.

If $F \rightarrow E \rightarrow X$ and $F \rightarrow E_{M} \rightarrow X$ were principal $F$-bundles in the original sense of Dold and Lashof, we could finish the proof by merely quoting 5.2 and 5.3 [5]. Fortunately, 5.2 and 5.3 are still true with our new definition of $F$-bundles, except that the maps in the bases are not the identities, but instead homotopic to the identities. More precisely, we state

5.2' Proposition. Let $F \rightarrow E^{i} \rightarrow X$ be principal $F$-bundles (see 3.6) for $i=0,1,2$, with a $\mathrm{CW}$-complex $X$ as common base, and with $F$ a connected $\mathrm{CW} H$-space as common fiber.

Suppose we have the commutative diagram

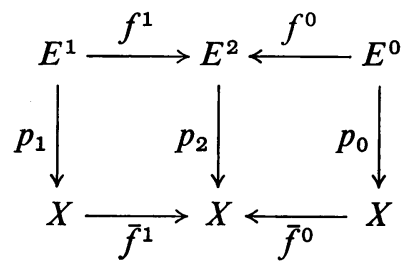


where $f^{1}$ and $f^{0}$ are principal maps. Let $\bar{f}^{t}$ be a homotopy between $\bar{f}^{0}$ and $\bar{f}^{1}$. Then there exists a principal map $f: E^{0} \rightarrow E^{1}$ lying over a map which is homotopic to the identity.

$f$ can be chosen so that $f^{0} \simeq f^{1} f$ by a principal homotopy $D^{t}: E^{0} \rightarrow E^{1}$ and $p D^{t} \simeq \bar{f}^{t} p^{0}$.

5.3' Corollary. Let $F \rightarrow E^{1} \rightarrow X$ and $F \rightarrow E^{0} \rightarrow X$ be principal F-bundles with $X$ and $F \mathrm{CW}$-spaces and $F$ connected. Let $g: E \rightarrow E^{0}$ be a principal map lying over a map which is homotopic to the identity map. Then there exists a principal map $f: E^{0} \rightarrow E^{1}$ such that $f g \cong \mathrm{id}$ and $g f \cong \mathrm{id} . B y \cong$ we shall mean there exists a principal homotopy between the two maps $D^{t}$ such that for each value of $t, D^{t}$ lies over a map homotopic to the identity.

The proof of $5.3^{\prime}$ is now the same as the proof of 5.3 [5] since we have made the necessary modifications in the meanings of principal bundle and $\cong$.

We shall now indicate the necessary modifications in the proof of 5.2.

Take a cellular subdivision of $X$ so fine that each cell $V$ is contained in some neighborhood $U$ over which the bundles are "almost" trivial. We now start at the top of p. 299.

Let $x^{0}$ be a vertex of $B . i: H \rightarrow p^{-1}\left(x_{0}\right), x_{0} \in U$ for some neighborhood $U$ over which the bundles are "almost" trivial. Then we have the maps $s_{i}: U \rightarrow p_{i}^{-1}(U)$, $p_{i} s_{i}=\mathrm{id}$, and

$$
U \times F \stackrel{\psi_{U}^{i}}{\longrightarrow} p_{i}^{-1}(U) \stackrel{\varphi_{U}^{i}}{\longrightarrow} U \times F
$$

for $i=0,1,2$. Consider the map $F \stackrel{s}{\rightarrow} p_{0}^{-1}\left(x_{0}\right)$ defined by $j(e)=s\left(x_{0}\right)$ where $e$ is the unit in $\mathrm{F}$, and $j(f)=s_{0}\left(x_{0}\right) \cdot f$ for all $f \in F$.

We also have the map $i: p_{0}^{-1}\left(x_{0}\right) \rightarrow F$ defined by the composition

$$
p_{0}^{-1}\left(x_{0}\right) \longrightarrow p_{0}^{-1}(U) \stackrel{\varphi_{U}^{0}}{\longrightarrow} U \times F \longrightarrow F
$$

where $U \times F \rightarrow F$ is projection onto the second factor. Consider the diagram

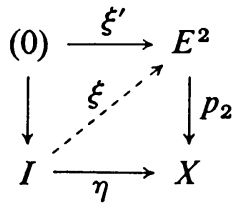

$$
\begin{aligned}
& \xi^{\prime}(0)=f^{0}(j(e)), \quad \eta(t)=\bar{f}^{t}\left(x_{0}\right) .
\end{aligned}
$$

Then $\xi^{\prime}$ has an extension to a map $\xi$ and $p \xi \simeq \eta$. Furthermore, we may assume $p \xi(1)=\eta(1)$.

The lifting $\xi$ may be chosen by covering the path $\eta(t)$ with neighborhoods over which we may take coverings lying over the segments of $\eta(t)$ in the neighborhoods. Since we do not have transition functions, we cannot patch together the liftings 
over each neighborhood. However, by choosing points in the intersections of the neighborhoods, and since the fiber is connected, we can patch together these partial liftings by running along the fibers.

Since $f_{*}^{1}: \pi_{0}\left(p_{1}^{-1}\left(x_{0}\right)\right) \rightarrow \pi_{0}\left(p_{2}^{-1}\left(\bar{f}^{1}\left(x_{0}\right)\right)\right)$ is an isomorphism, $\xi(1)$ can be assumed to be $f^{1}\left(y_{1}\right)$ for some $y_{1} \in E^{1}$. (At least it is connected to $f^{1}\left(y_{1}\right)$ by a path in the fiber.) Then we define $f: p_{0}^{-1}\left(x_{0}\right) \rightarrow E^{1}$ by $f(y)=y^{1} \cdot i(y)$.

Define $H(y, t)=\xi(t) \cdot i(y) \in E^{1}$.

Then $H(y, 1)=\xi(1) \cdot i(y)=f^{1}\left(y_{1}\right) \cdot i(y)=f^{1}\left(y_{1} i(y)\right)=f^{1} f(y)$.

$$
H(y, 0)=\xi(0) \cdot i(y)=f^{0}(j(e) \cdot i(y)) .
$$

But since

$$
p_{0}^{-1}(U) \stackrel{\varphi_{U}^{0}}{\longrightarrow} U F \stackrel{\psi_{U}^{0}}{\longrightarrow} p_{0}^{-1}(U)
$$

is homotopic to the identity, we have $y \simeq j(e) \cdot i(y)$.

Hence we connect $f^{0}(j(e) \cdot i(y))$ to $f^{0}(y)$ by a principal homotopy. Thus we have $D: p_{0}^{-1}\left(x_{0}\right) \rightarrow E^{1}$ a principal homotopy between $f^{0}$ and $f^{1} f$, and we have $p_{1} D^{t} \simeq$ $f^{t} p_{0}$. Let $f$ and $D$ be defined over the $n$-skeleton of $X$.

The inductive step follows in a corresponding manner. That is, one identifies $\left(p_{0}\right)^{-1}(V \times F)$ with $V \times F$ by means of the map $\psi_{V}^{0}$. One has the homotopy commutative diagram

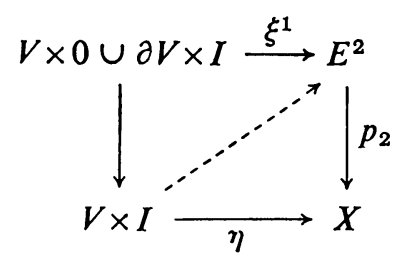

where

$$
\begin{aligned}
\xi^{1}(x, 0) & =f^{0}\left(\psi_{V}^{0}(x, e)\right) \quad \text { if } X \in V, \\
\xi^{1}(z, t) & =D^{t}\left(\psi_{V}^{0}(z, e)\right) \quad \text { if } z \in S=\partial V, \\
\eta(x, t) & =\bar{f}^{t}(z) .
\end{aligned}
$$

We have a homotopy commutative diagram and by the ACHEP, we can extend $\xi^{1}$ to a map $d: V \times I \rightarrow E^{2}$ and $p d \simeq \eta$.

It is now easy to finish the proof, using exactly the arguments on p. 299.

Now we restate the classification theorem as follows:

6.10 Theorem. Let $X$ be a countable $\mathrm{CW}$-space and let $F$ be a countable $\mathrm{CW}$ $H$-space (associative). There is a one-to-one correspondence between equivalence classes of principal F-bundles over $X$ and homotopy classes of maps from $X$ to $B_{\infty}(F)$.

Proof. We have just proved that there is a one-to-one correspondence between equivalence classes of principal $F$-bundles over $X$ with fiber $F$ and SHM homotopy classes of SHM classes of maps from $\Omega X$ to $F$. 
But there is a one-to-one correspondence between SHM homotopy classes of maps from $(\Omega X)$ to $(F)$, and homotopy classes of maps from $B_{\infty}(\Omega X)$ to $B_{\infty}(F)$. Since $B_{\infty}(\Omega X) \sim X$, we have finished.

7. Classifying fiber homotopy equivalence. Dold and Lashof $[5, \S 7$ in particular] have shown how to classify fiber bundles with respect to homotopy equivalence in terms of principal fiberings. Briefly, let $F \rightarrow E \stackrel{p}{\rightarrow} X$ and $F \rightarrow E^{\prime} \stackrel{p^{\prime}}{\rightarrow} X^{\prime}$ be fiber bundles. (Here a fiber bundle is taken in the sense of Steenrod [14] but not necessarily with structure group.) A fiberwise map



is admissible if for every $x \in X, f_{x}: p^{-1}(x) \rightarrow p^{\prime-1}(f(x))$ is a homotopy equivalence where $f_{x}$ is $f$ restricted to the fiber $p^{\prime}(x)$ over $x$. A homotopy $d: E \times I \rightarrow E^{\prime}$ is admissible if $d_{t}$ is admissible for each $t \in I\left(d_{t}(y)=d(y, t)\right)$. Now let $X=X^{\prime}$. A fiber homotopy equivalence between $E \rightarrow X$ and $E^{\prime} \rightarrow X$ is a pair of admissible maps
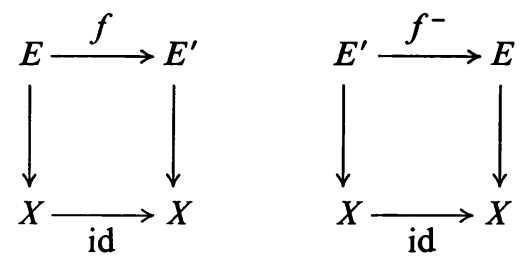

such that $f f^{-} \simeq$ id and $f^{-} f \simeq$ id, both homotopies being admissible homotopies leaving the base space fixed.

Now let $F \rightarrow E \stackrel{p}{\rightarrow} X$ be a fiber bundle (in the above sense) with locally compact fiber $F$. Let $H$ be the space of all homotopy equivalences from $F$ to $F$ (compactopen topology). Composition of maps forms a multiplication turning $H$ into an associative $H$-space. Let $\tilde{E}$ be the set of all continuous mappings $\varphi: F \rightarrow E$ (compact-open topology) such that $\varphi(F)$ is contained in some fiber $p^{-1}(x)$ and $\varphi: F \rightarrow p^{-1}(x)$ is a homotopy equivalence. $H \rightarrow \widetilde{E} \stackrel{\tilde{p}}{\rightarrow} X$ is a principal $H$-bundle. $\tilde{p} \varphi=p \varphi(F) . H \rightarrow \widetilde{E} \stackrel{\tilde{p}}{\rightarrow} X$ is the principal $H$-bundle associated to the fiber bundle $F \rightarrow E \stackrel{p}{\rightarrow} B$.

Dold and Lashof have proved:

THEOREM 7.1. Two fiber bundles $F \rightarrow E \stackrel{p}{\rightarrow} X$ and $F \rightarrow E^{\prime} \stackrel{p^{\prime}}{\rightarrow} X$ over $X$ with the same locally compact fiber are fiber homotopy equivalent if and only if their associated principal H-bundles are equivalent (in the sense of principal bundle equivalence). 
Let $L F(X)$ be the fiber homotopy equivalence of fiber spaces $p: E \rightarrow X$ with fibers of the homotype of $F$. Here fiber space is in the sense of [12, Definition 2.1]. That is, we are not assuming any local triviality, and homotopies may be vertically lifted. Then Stasheff (Classification Theorem 1) has proved $L F(X)=X, B(H(F))$. Using this and our classification theorem, we see that there is a one-to-one correspondence between equivalence classes of principal $H(F)$-bundles (in the modified sense) and fiber homotopy equivalences of fiber spaces over $X$ with fiber $F$.

8. $n$-equivalence and the homotopy type of $B_{n}(X)$.

8.1 The homotopy equivalence of $X$ and $B_{\infty}(\Omega X)$.

8.1.1 TheOREM. Let $X$ be any space. Then there exists a map $\varphi: B_{\infty}(\Omega X) \rightarrow X$ such that $H_{*}(\varphi): H_{*}\left(B_{\infty}(\Omega X)\right) \rightarrow H_{*}(X)$ is an isomorphism of singular homology. If $X$ is a countable CW-complex then $\varphi$ is a homotopy equivalence.

(The proof given here is that of Theorem 9.1 (Stasheff [13, p. 305]), and is included here for the sake of completeness.)

Proof. Define inductively

$$
\varphi_{i}: B_{i-1}(\Omega X) \rightarrow X, \quad \psi_{i}: E_{i-1}(\Omega X) \rightarrow \Lambda X, \quad \theta_{i}: C\left(E_{i-1}(\Omega X)\right) \rightarrow \Lambda X,
$$

where $\Lambda X$ is the path space of $X$ and $C\left(E_{i-1}(\Omega X)\right)$ is the cone of $E_{i-1}(\Omega X)$ as follows:

Let $\psi_{1}: \Omega X \rightarrow \Lambda X$ be the inclusion. Then the formulas

$$
\begin{aligned}
\theta_{i}(t, z)(s) & =\psi_{i}(z)(t s) \quad \text { for } z \in E_{i-1}(\Omega X), \\
\varphi_{i}(t, z) & =\pi \theta_{i}(t, z), \\
\psi_{i+1}(z|t| \lambda) & \left.=\theta_{i}(t, z) \cdot \lambda \quad \text { (the usual action of } \Omega X \text { on } \Lambda X\right)
\end{aligned}
$$

define $\theta_{i}, \varphi_{i}, \psi_{i+1}$ inductively for all $i$, inducing $\varphi: B_{\infty}(\Omega X) \rightarrow X$ and $\psi: E_{\infty}(\Omega X) \rightarrow$ $\Lambda X$. Then the diagram

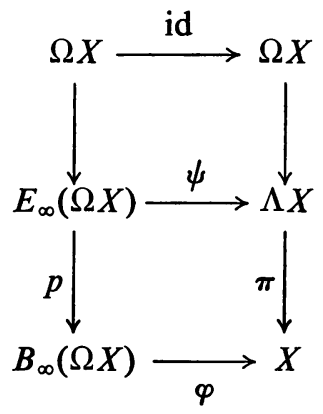

is commutative, and respects the action of $\Omega X$ on $p$ and $\pi$.

8.2 The strongly homotopy multiplicative equivalence of $\Omega B_{\infty}(X)$ and $X$.

8.2.1 Theorem. Let $X$ be any $H$-space. Let $n \geqq 2$ be an integer. Then there is an $(n-1)-H M$ map from $X$ to $\Omega\left(B_{n}(X)\right)$. 
Proof. We first remark that Sugawara [16, p. 262] proves (Lemma 4.1) that there exists a SHM map from $X$ to $\Omega B$ for a principal universal bundle $p: E \rightarrow B$ for the $H$-space $X$. He inductively defines, for $\left(t_{1}, \ldots, t_{n}\right) \in \partial I^{n}, N_{n}\left(x_{0}, t_{1}, \ldots, t_{n}, x_{n}\right)$ $\in \Omega B$, and $\tilde{N}_{n}\left(x_{0}, t_{1}, \ldots, t_{n}, x_{n}\right) \in \Lambda E$ covering $N_{n}\left(x_{0}, t_{1}, \ldots, t_{n}, x_{n}\right)$. Then using the contractibility of $\Lambda E, \tilde{N}_{n}\left(x_{0}, t_{1}, \ldots, t_{n}, x_{n}\right)$ is defined for all $\left(t_{1}, \ldots, t_{n}\right) \in I^{n}$ and hence $N_{n}\left(x_{0}, t_{1}, \ldots, t_{n}, x_{n}\right)$ is also defined. This type of argument could be used to prove 8.2.1. That is, one could construct $\tilde{N}_{i}\left(x_{0}, \ldots, t_{i}, x_{i}\right) \in \Lambda E_{i}(X)$ covering $N_{i}\left(x_{0}, t_{1}, \ldots, t_{i}, x_{i}\right)$ for values of $\left(t_{1}, \ldots, t_{i}\right) \in \partial I^{i}$, and then using the fact that $E_{i}(X)$ is contractible in $E_{i+1}(X)$, extend $\tilde{N}_{i}$ to be defined for all values of $\left(t_{1}, \ldots, t_{n}\right)$. However, it is possible to define $\left\{N_{0}, N_{1}, \ldots, N_{n-1}\right\}$ directly, which is done here.

Define $K_{0}: X \rightarrow \Lambda E_{1}(X) \subset \Lambda E_{n}(X)$ by letting, for each $x \in X, K_{0}(x)$ be that path in $E_{1}(X)$ of length 1 given by $K_{0}(x)(s)=q(x, s, e)$ if $s \in I$. (Recall that $q(x, s, e)$ is the class in $E_{1}(X)$ represented by $(x, s, e)$.)

In general, for $1 \leqq m \leqq n-1$, define $K_{m}: X_{m} \rightarrow \Lambda E_{m+1}(X) \subset \Lambda E_{n}(X)$ by letting, for each $\omega \in X_{m}, K_{m}(\omega)$ be that path of length $1+t_{1}(\omega)+\cdots+t_{m}(\omega)$ given by

$$
\begin{aligned}
K_{m}(\omega)(s)= & q\left(x_{0}(\omega), 1-t_{1}(\omega), \ldots, 1-t_{m}(\omega), X_{m}(\omega), s, e\right) \quad \text { if } 0 \leqq s \leqq 1, \\
= & q\left(x_{0}(\omega), 1-t_{1}(\omega), \ldots, 1-t_{m-1}(\omega), s-t_{m}(\omega), x_{m}(\omega)\right) \\
= & q\left(x_{0}(\omega), 1-t_{1}(\omega), \ldots, 1-t_{i-1}(\omega), x_{i-1}(\omega),\right. \\
& \left.s-\left(t_{m}(\omega)+t_{m-1}(\omega)+\cdots+t_{i}(\omega)\right), x_{i}(\omega) \cdots x_{m}(\omega)\right) \\
& \text { if } 1+t_{i+1}(\omega)+\cdots+t_{m}(\omega) \leqq s \leqq 1+t_{i}(\omega)+\cdots+t_{m}(\omega) \\
& \text { and } Z \leqq i \leqq m-1, \\
= & q\left(x_{0}(\omega), s-\left(t_{1}(\omega)+\cdots+t_{m}(\omega)\right), x_{1}(\omega) \cdots x_{m}(\omega)\right) \\
& \quad \text { if } 1+t_{2}(\omega)+\cdots+t_{m}(\omega) \leqq s \leqq 1+t_{1}(\omega)+\cdots+t_{m}(\omega) .
\end{aligned}
$$

Then define $N_{m}: X_{m} \rightarrow \Omega B_{n}(X)$ by defining $N_{m}=\Lambda p K_{m}$ where $\Lambda E_{n}(X) \stackrel{\Lambda_{p}}{\rightarrow} \Lambda B_{n}(X)$ is induced by the projection $p: E_{n}(X) \rightarrow B_{n}(X)$.

8.2.2 TheOREM. Let $X$ be a CW H-space. Let $n>1$ be an integer. Let $N=$ $\left\{N_{0}, N_{1}, \ldots, N_{n-1}\right\}$ be the (n-1)-HM map from $X$ to $\Omega B_{n}(X)$ constructed in 8.2.1. Let $\left\{M_{0}, M_{1}, \ldots, M_{i}\right\}$ be the $\infty-H M$ map from $\Omega B_{n}(X)$ to $X$ for the principal quasifibering $X \rightarrow E_{n}(X) \rightarrow B_{n}(X)$ constructed in 5.3. Then the (n-1)-HM map $\left\{M_{0}, M_{1}, \ldots, M_{n-1}\right\} \cdot\left\{N_{0}, N_{1}, \ldots, N_{n-1}\right\}$ is homotopic to the $(n-1)-H M$ map $\left\{I_{0}, I_{1}, \ldots, I_{n-1}\right\}$ formed by the identity map of $X$.

$$
X \stackrel{N}{\longrightarrow} \Omega B_{n}(X) \stackrel{M}{\longrightarrow} X .
$$

Proof. (The proof is very similar to the proof of 5.3.) Recall that $X_{n}=X \times$ $(J \times X)^{n}$ where $J=[0,2]$ and $I_{i}: X_{i} \rightarrow X$ is given by $I_{i}(\omega)=x_{0}(\omega) \cdots x_{i}(\omega)$. Also recall that in 5.3 the maps $\left\{M_{0}, \ldots, M_{i}, \ldots\right\}$ were constructed in terms of maps $\left\{\xi_{0}, \xi_{1}, \ldots, \xi_{n}, \ldots\right\}=\left\{\bar{H}_{0}, \bar{H}_{1}, \ldots, \bar{H}_{n}, \ldots\right\}$. Let

$$
\begin{gathered}
K=I \times X, \quad L=\partial I \times X, \\
M=I \times X \times \partial I, \quad M^{\prime}=M \cap(K \times(0) \cup L \times I),
\end{gathered}
$$


and define maps $\psi_{0}^{\prime}, \nu_{0}$,

$$
\begin{aligned}
& \left(K \times(0) \cup L \times I, M^{\prime}\right) \stackrel{\psi_{0}^{\prime}}{\longrightarrow}\left(E_{n}(X), X\right) \\
& \psi_{0}^{\prime}(t, x, s)=e \quad \text { if } s=0, \\
& =K_{0}(x)(s) \text { if } t=1 \text { and } s \in I \text {, } \\
& =\xi_{0}\left(N_{0}(x), s\right) \quad \text { if } t=0 \text { and } s \in I \text {. }
\end{aligned}
$$

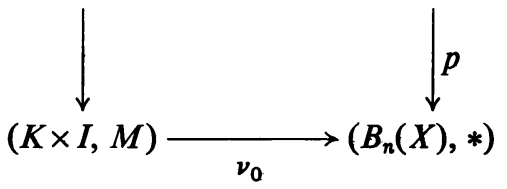

$\nu_{0}(t, x, s)=N_{0}(x)(s)$.

Now by 5.2 there is a homotopy $Y_{0}:\left(\Omega B_{n}(X) \times I \times I, \Omega B_{n}(X) \times \partial I \times I\right) \rightarrow$ $\left(B_{n}(X), *\right)$ such that

$$
\begin{aligned}
Y_{0}(\lambda, s, u) & =p \xi_{0}(\lambda, s) & & \text { if } u=0 \\
& =\lambda(|\lambda| \cdot s) & & \text { if } u=1, \\
& =* & & \text { if } s \in \partial I .
\end{aligned}
$$

Thus there is a homotopy $v_{0}: p \psi_{0}^{\prime} \simeq \nu_{0} \mid K \times(0) \cup L \times I$, that is

is given by

$$
v_{0}:\left((K \times(0) \cup L \times I) \times I, M^{\prime} \times I\right) \rightarrow\left(B_{n}(X), *\right)
$$

$$
\begin{aligned}
v_{0}(t, x, s, z) & =Y_{0}\left(N_{0}(x), s, z\right) & & \text { if } t=0,0 \leqq z \leqq 1, \\
& =N_{0}(x)(s) & & \text { if } t=1,0 \leqq x \leqq 1, \\
& =e & & \text { if } s=0 .
\end{aligned}
$$

Then by the ACHEP for quasifiberings, since $X$ is a CW-complex, $\psi_{0}^{\prime}$ extends to $\psi_{0}$ and the homotopy $v_{0}$ extends to $V_{0}:(K \times I \times I, K \times \partial I \times I) \rightarrow\left(B_{n}(X), *\right)$ so that $V_{0}: p \psi_{0} \simeq \nu_{0}$.

Suppose inductively that $\psi_{0}, \psi_{1}, \ldots, \psi_{m-1}$ are defined for $1 \leqq i \leqq m-1$ so that $\psi_{i}:\left(I \times X_{i} \times I, I \times X_{i} \times \partial I\right) \rightarrow\left(E_{n}(X), X\right)$ satisfies

$$
\begin{aligned}
\psi_{i}\left(t_{0}, w, s\right) & =e \text { if } s=0, \\
& =\psi_{i-1}\left(t_{0}, \delta_{j}(\omega), s\right) \quad \text { if } t_{j}(\omega)=0, \\
& =\psi_{i-j}\left(t_{0}, \omega(j, i), 2 s\right) \quad \text { if } t_{j}(\omega)=1 \text { and } 0 \leqq s \leqq \frac{1}{2}, \\
& =\psi_{j-i}\left(t_{0}, \omega(0, j-1), 2\left(s-\frac{1}{2}\right)\right) \cdot \psi_{i-j}\left(t_{0}, \omega(j, i), 1\right) \quad \text { if } t_{j}(\omega)=1 \\
& \quad \text { and } \frac{1}{2} \leqq s \leqq 1, \\
& =K_{i}(\omega)(s) \quad \text { if } t_{0}=1(\operatorname{see} I V .2 .1),
\end{aligned}
$$

We will call the above equation (1). 
Let $\nu_{i}:\left(I \times X_{i} \times I, I \times X_{i} \times \partial I\right) \rightarrow\left(B_{n}(X), *\right)$ be given by

$$
\nu_{i}\left(t_{0}, \omega, s\right)=\bar{N}_{i}(\omega, s) \quad\left(=N_{i}(\omega, s) \text { normalized }\right) .
$$

Also assume $V_{i}:\left(I \times X_{i} \times I, I \times X_{i} \times \partial I\right) \rightarrow\left(B_{i}(X), *\right)$ is a homotopy between $p \psi_{i}$ and $\nu_{i}$ satisfying

$$
\begin{aligned}
V_{i}\left(t_{0}, \omega, s, u\right) & =V_{i-1}\left(t_{0}, \delta_{j}(\omega), s, u\right) \quad \text { if } t_{j}(\omega)=0 \\
& =V_{i-j}\left(t_{0}, \omega(j, i), 2 s, u\right) \text { if } t_{j}(\omega)=1 \text { and } 0 \leqq s \leqq \frac{1}{2} \\
& =V_{j-1}\left(t_{0}, \omega(0, j-1), 2\left(s-\frac{1}{2}\right), u\right) \text { if } t_{j}(\omega)=1 \text { and } \frac{1}{2} \leqq s \leqq 1 .
\end{aligned}
$$

Now let $K=I \times X_{i}, L=\partial I \times X_{i} \cup I \times X_{i} \cap\left\{\omega \mid t_{i}(\omega) \in \partial I\right.$ for some $\left.i\right\} . M=K \times \partial I$, $M^{\prime}=(K \times(0) \cup L \times I) \cap M$. Then

$$
\psi_{m}^{\prime}:\left(K \times 0 \cup L \times I, M^{\prime}\right) \rightarrow\left(E_{n}(X), X\right)
$$

is determined by equation (1). Equation (3) determines a homotopy

$$
\begin{gathered}
v_{m}:\left(K \times 0 \cup L \times I \times I, M^{\prime} \times I\right) \rightarrow\left(B_{n}(X), *\right), \\
v_{m}: p \psi_{m}^{\prime} \simeq v_{m} \mid K \times(0) \cup L \times I .
\end{gathered}
$$

That is, by definition

$$
\begin{aligned}
v_{m}\left(t_{0}, \omega, s, u\right) & =v_{m-1}\left(t_{0}, \delta_{i}(\omega), s, u\right) \text { if } t_{i}(\omega)=0, \\
& =V_{m-i}\left(t_{0}, \omega(0, i-1), 2\left(s-\frac{1}{2}\right), u\right) \text { if } t_{i}(\omega)=1 \text { and } 0 \leqq s \leqq \frac{1}{2}, \\
& =\nu_{m}(\omega, s) \text { if } t_{0}=1, \\
& =y_{m}(\omega, s, u) \text { if } t_{0}=0 \text { and } 0 \leqq s \leqq \frac{1}{2},
\end{aligned}
$$

where in the above equation, $y_{m}$ is the homotopy between $p \xi_{m}$ and $\eta$ given in 5.2 , that is

$$
\begin{aligned}
y_{m}(\omega, s, 0) & =p \xi_{k}\left(z_{0}, 2\left(t_{j}(\omega)-\frac{1}{2}\right), \ldots, 2\left(t_{j_{k}}(\omega)-\frac{1}{2}\right), z_{k}, s\right), \\
y_{m}(\omega, s, 1) & =\eta\left(z_{0}, 2\left(t_{j}(\omega)-\frac{1}{2}\right), \ldots, 2\left(t_{j_{k}}(\omega)-\frac{1}{2}\right), z_{k}, s\right) \\
& =\left(z_{0} \cdots z_{k}\right)\left(s \cdot\left|z_{0} \cdots z_{k}\right|\right)=\nu_{m}(\omega, s) .
\end{aligned}
$$

Then by the ACHEP $\psi_{m}^{\prime}$ extends to $\psi_{m}$ and $v_{m}$ extends to $V_{m}: p \psi_{m} \simeq \nu_{m}$, giving the homotopy commutative diagram

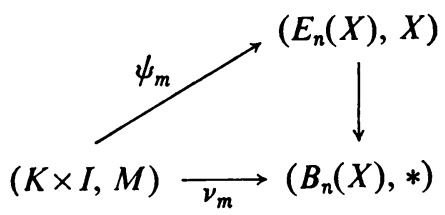

This completes the inductive definition of $\psi_{0}, \psi_{1}, \ldots, \psi_{n-1}$. Then $h=\left\{h_{0}, h_{1}, \ldots\right.$, $\left.h_{n-1}\right\}$ is a homotopy between $\left\{I_{0}, I_{1}, \ldots, I_{n-1}\right\}$ and $\left\{M_{0}, M_{1}, \ldots, M_{n-1}\right\} \cdot\left\{N_{0}\right.$, $\left.N_{1}, \ldots, N_{n-1}\right\}$ where $h_{i}\left(t_{0}, \omega, s\right)=\psi_{i}\left(t_{0}, \omega, 1\right)$. 
8.2.3 Theorem. Let $X$ be a CW H-space. Let $\left\{N_{0}, N_{1}, \ldots N_{i}, \ldots\right\}$ be the $\infty$-HM map from $X$ to $\Omega B_{\infty}(X)$ constructed in 8.2.1. Let $\left\{M_{0}, M_{1}, \ldots, M_{i}, \ldots\right\}$ be the $\infty-H M$ map from $\Omega B_{\infty}(X)$ to $X$ for the principal universal quasifibering $X \rightarrow E_{\infty}(X) \rightarrow B_{\infty}(X)$ constructed in 5.3. Then the composition $\left\{P_{0}, P_{1}, \ldots, P_{i}, \ldots\right\}$ $=\left\{N_{0}, N_{1}, \ldots, N_{i}, \ldots\right\} \cdot\left\{M_{0}, M_{1}, \ldots, M_{i}, \ldots\right\}$ is homotopic to the $\infty-H M$ map $\left\{I_{0}, I_{1}, \ldots, I_{i}, \ldots\right\}$ formed by the identity of $\Omega B_{\infty}(X)$,

$$
\Omega B_{\infty}(X) \stackrel{M}{\longrightarrow} X \stackrel{N}{\longrightarrow} \Omega B_{\infty}(X) .
$$

Proof. Recall that by 5.1 there is a mapping, $\xi_{0}: \Omega B_{\infty}(X) \times I \rightarrow E_{\infty}(X)$ so that

$$
\begin{aligned}
\xi_{0}(\lambda, s) & =M_{0}(\lambda) & & \text { if } s=1, \\
& =e & & \text { if } s=0,
\end{aligned}
$$

and there is a homotopy

$$
Y_{0}:\left(\Omega B_{\infty}(X) \times I \times I, \Omega B_{\infty}(X) \times \partial I \times I\right) \rightarrow\left(B_{\infty}(X), *\right)
$$

so that

$$
\begin{aligned}
Y_{0}(\lambda, s, u) & =p \xi_{0}(\lambda, s) & & \text { if } u=0, \\
& =\lambda(s \cdot|\lambda|) & & \text { if } u=1 .
\end{aligned}
$$

Define $\varphi_{0}^{\prime}: I \times \Omega B_{\infty}(X) \times \partial I \cup \partial \mathrm{I} \times \Omega B_{\infty}(X) \times I \rightarrow E_{\infty}(X)$ by

$$
\begin{aligned}
\varphi_{0}^{\prime}\left(t_{0}, \lambda, s\right) & =M_{0}(\lambda) & & \text { when } s=1, \\
& =e & & \text { when } s=0 \\
& =\xi_{0}(\lambda, s) & & \text { when } t_{0}=0 \\
& =K_{0}\left(M_{0}(\lambda)(s)\right) & & \text { when } t_{0}=1 .
\end{aligned}
$$

(Recall that $\left\{K_{0}, \ldots, K_{n}, \ldots\right\}$ was defined in 8.2.1.) Since $E_{\infty}(X)$ is contractible, $\varphi_{0}^{\prime}$ extends to $\varphi_{0}: I \times \Omega B_{\infty}(X) \times I \rightarrow E_{\infty}(X)$. Then $p \varphi_{0}$ provides a homotopy between $p \xi_{0}(\lambda, s)$ and $N_{0} M_{0}(\lambda)(s)$. Since $p \xi_{0}(\lambda, s) \simeq \lambda(s \cdot|\lambda|)$, we get $\lambda(s) \simeq$ $N_{0} M_{0}(\lambda)(s)$. More precisely, define $h_{0}: I \times \Omega B_{\infty}(X) \rightarrow \Omega B_{\infty}(X)$ by

$$
\begin{array}{rlrl}
h_{0}\left(t_{0}, \lambda\right) & =W_{0}\left(\lambda, s, 2 t_{0}\right) & & \text { if } 0 \leqq t_{0} \leqq \frac{1}{2}, \\
& =\psi_{0}\left(2\left(t_{0}-\frac{1}{2}\right), \lambda, s\right) & \text { if } \frac{1}{2} \leqq t_{0} \leqq 1,
\end{array}
$$

where $W_{0}(\lambda, s, t)=Y_{0}(\lambda, s /|\lambda|, t)$ if $|\lambda| \neq 0$ and $\psi_{0}: I \times \Omega B_{\infty}(X) \rightarrow \Omega B_{\infty}(X)$ is defined by $\psi_{0}\left(t_{0}, \lambda\right)(s)=p \varphi_{0}\left(t_{0}, \lambda, s\right)$.

In general, $h_{n}$ is defined in terms of $W_{n}$ and $\psi_{n}$ as follows. $h_{n}: I \times\left(\Omega B_{\infty}(X)\right)_{n} \rightarrow$ $\Omega B_{\infty}(X)$ is given by

$$
\begin{aligned}
h_{n}\left(t_{0}, \omega, s\right) & =W_{n}\left(\omega, s \cdot \omega, 2 t_{0}\right) & & \text { if } 0 \leqq t_{0} \leqq \frac{1}{2} \\
& =\psi_{n}\left(2\left(t_{0}-\frac{1}{2}\right), \omega, s\right) & & \text { if } \frac{1}{2} \leqq t_{0} \leqq 1,
\end{aligned}
$$

where $W_{n}$ is the homotopy between $p H_{n}(\omega, s)$ and $\lambda_{0}(\omega) \cdots \lambda_{n}(\omega)(s)$ defined in 5.2 and $\psi_{n}$ is defined inductively in a way similar to the way that $\psi_{0}$ was defined. That is, having defined $\varphi_{0}, \varphi_{1}, \ldots, \varphi_{n-1}$, where

$$
\varphi_{i}:\left(I \times\left(\Omega B_{\infty}(X)\right)_{i} \times I, I \times\left(\Omega B_{\infty}(X)\right)_{i} \times \partial I\right) \rightarrow\left(E_{\infty}(X), X\right),
$$


define inductively

$$
\varphi_{n}^{\prime}: I \times L \times I \cup \partial I \times\left(\Omega B_{\infty}(X)\right)_{n} \times I \cup I \times\left(\Omega B_{\infty}(X)\right)_{n} \times \partial I \rightarrow E_{\infty}(X)
$$

by

$$
\begin{aligned}
\varphi_{n}^{\prime}\left(t_{0}, \omega, s\right) & =e \quad \text { if } s=0, \\
& =\varphi_{n-1}\left(t_{0}, \delta_{i}(\omega), s\right) \quad \text { if } t_{i}(\omega)=0, \\
& =\varphi_{n-i}(\omega(i, n), 2 s) \quad \text { if } t_{i}(\omega)=1 \text { and } 0 \leqq s \leqq \frac{1}{2} \\
& =\varphi_{i-1}\left(\omega(0, i-1), 2\left(s-\frac{1}{2}\right)\right) \cdot \varphi_{n-i}(\omega(i, n), 1) \quad \text { if } t_{i}(\omega)=1 \text { and } \frac{1}{2} \leqq s \leqq 1, \\
& =\xi_{n}(\omega, s) \text { if } t_{0}=0, \\
& =\bar{K}_{k}\left(z_{0}, 2\left(t_{j_{1}}(\omega)-\frac{1}{2}\right), \ldots, 2\left(t_{j_{k}}(\omega)-\frac{1}{2}\right), z_{j_{k}}\right)(s) \quad \text { if } t_{0}=1, \\
& =\bar{K}_{k}\left(z_{0}, 2\left(t_{j_{1}}(\omega)-\frac{1}{2}\right), \ldots, 2\left(t_{j_{k}}(\omega)-\frac{1}{2}\right), z_{j_{k}}\right)(1) \text { if } s=1,
\end{aligned}
$$

where, as before, $z_{v}=M_{j_{v}+1-j_{v-1}}\left(\omega\left(j_{v}, j_{v+1}-1\right)\right)$ and $\frac{1}{2} \leqq t_{i}(\omega) \leqq 1$ when $i=j_{1}, \ldots, j_{k}$ and $0 \leqq t_{i}(\omega) \leqq \frac{1}{2}$ when $i \neq j_{1}, \ldots, j_{k}$. Also we recall that in the above formula, $L=\Omega B_{\infty}(X) \cap\left\{\omega \mid t_{i}(\omega) \in \partial I\right.$ for some $\left.i\right\} . \xi_{n}$ is defined in 5.2, $\bar{K}_{k}$ is $K_{k}$ normalized, and $\left\{K_{0}, \ldots, K_{i}, \ldots\right\}$ is defined in 8.2.1.

Since $E_{\infty}(X)$ is contractible, $\varphi_{n}^{\prime}$ extends to

$$
\varphi_{n}:\left(I \times\left(\Omega B_{\infty}(X)\right)_{n} \times I, I \times\left(\Omega B_{\infty}(X)\right)_{n} \times \partial I\right) \rightarrow\left(E_{\infty}(X), X\right) .
$$

Then $\psi_{n}: I \times \Omega B_{\infty}(X) \rightarrow \Omega B_{\infty}(X)$ is defined by

$$
\psi_{n}\left(t_{0}, \omega\right)(s)=p \varphi_{n}\left(t_{0}, \omega, s\right), \quad 0 \leqq s \leqq 1 .
$$

This completes the inductive definition of $\left\{\psi_{0}, \ldots, \psi_{n}, \ldots\right\}$ and $\left\{h_{0}, \ldots, h_{n}, \ldots\right\}$. Then $\left\{h_{0}, h_{1}, \ldots, h_{n}, \ldots\right\}$ forms a homotopy between $\left\{P_{0}, P_{1}, \ldots, P_{n}, \ldots\right\}$ and $\left\{I_{0}, I_{1}, \ldots, I_{n}, \ldots\right\}$.

Combining Theorem 8.2.3 with 8.2.2 (with $n=\infty$ ), we get

8.2.4 Theorem. Let $X$ be a $\mathrm{CW} H$-space. Then $X$ is $\infty$-equivalent to $\Omega B_{\infty}(X)$.

8.3 The homotopy type of $B_{n}(X)$.

8.3.1 THEOREM. Suppose $Y$ and $Z$ are n-equivalent $H$-spaces. Then $B_{n+1}(Y)$ and $B_{n+1}(Z)$ have the same homotopy type. (The case $n=1$ has been proved somewhat differently by Stasheff [11, p. 744].)

Proof. Since $Y$ and $Z$ are $n$-equivalent, there are $n$-HM maps $\left\{M_{0}, M_{1}, \ldots, M_{n}\right\}$ from $Y$ to $Z$ and $\left\{N_{0}, \ldots, N_{n}\right\}$ from $Z$ to $Y$ such that $\left\{M_{0}, \ldots, M_{n}\right\}\left\{N_{0}, \ldots, N_{n}\right\}$ and $\left\{N_{0}, \ldots, N_{n}\right\}\left\{M_{0}, \ldots, M_{n}\right\}$ are (n-HM) homotopic to the $n$-HM maps induced by the identity maps of $Z$ and $Y$. Writing $M=\left\{M_{0}, \ldots, M_{n}\right\}, N=\left\{N_{0}, \ldots, N_{n}\right\}$, and $I=\left\{I_{0}, \ldots, I_{n}\right\}$ where $I$ is the $n$-HM map from $Z$ to $Z$ 'induced by the identity map of $Z$,

$$
B_{n+1}(M) B_{n+1}(N) \simeq B_{n+1}(M N) \simeq B_{n+1}(I) \simeq \mathrm{id}_{B_{n+1}}(Z)
$$

Similarly $B_{n+1}(N) B_{n+1}(M) \simeq \operatorname{id}_{B_{n+1}}(Y)$.

8.3.2 THEOREM. Let $Y$ and $Z$ be $\mathrm{CW} H$-spaces such that $B_{n+1}(Y)$ and $B_{n+1}(Z)$ have the same homotopy type. Then $Y$ and $Z$ are n-equivalent. 
Proof. Let $f: B_{n+1}(Y) \rightarrow B_{n+1}(Z)$ and $g: B_{n+1}(Z) \rightarrow B_{n+1}(Y)$ be maps such that $f g \simeq$ id and $g f \simeq$ id. We construct $n$-HM maps from $Y$ to $Z$ and $Z$ to $Y$ as follows:

$$
Y \rightarrow \Omega B_{n+1}(Y) \rightarrow \Omega B_{n+1}(Z) \rightarrow Z, \quad Z \rightarrow \Omega B_{n+1}(Z) \rightarrow \Omega B_{n+1}(Y) \rightarrow Y .
$$

Since $Z \rightarrow E_{n+1}(Z) \rightarrow B_{n+1}(Z)$ is a principal quasifibering, by 5.3 there is a SHM map $M=\left\{M_{0}, M_{1}, \ldots, M_{n}\right\}$ from $\Omega B_{n+1}(Z)$ to $Z$. Similarly there is $N$ from $\Omega B_{n+1}(Y)$ to $Y$. By 8.2.1 there is an $n$-HM map $P=\left\{P_{0}, \ldots, P_{n}\right\}$ from $Y$ to $\Omega B_{n+1}(Y)$ and also $Q=\left\{Q_{0}, \ldots, Q_{n}\right\}$ from $Z$ to $\Omega B_{n+1}(Z)$. Theorem 8.2.2 showed that the compositions $N P$ and $M Q$ are $(n-\mathrm{HM})$ homotopic to the $(n-\mathrm{HM})$ maps from $Y$ to $Y$ and $Z$ to $Z$, respectively, induced by the identity maps of $Y$ and $Z$ respectively. $\Omega f: \Omega B_{n+1}(Y) \rightarrow \Omega B_{n+1}(Z)$ and $\Omega g: \Omega B_{n+1}(Z) \rightarrow \Omega B_{n+1}(Y)$ are multiplicative hence induce $n-\mathrm{HM}$ maps $T=\left\{\Omega f=T_{0}, T_{1}, \ldots, T_{n}\right\}$ and $U=$ $\left\{\Omega g=U_{0}, U_{1}, \ldots, U_{n}\right\}$ from $\Omega B_{n+1}(Y)$ to $\Omega B_{n+1}(Z)$ and from $\Omega B_{n+1}(Z)$ to $\Omega B_{n+1}(Y)$ respectively. The compositions of $n$-HM maps

$$
\begin{aligned}
& R: Y \stackrel{P}{\longrightarrow} \Omega B_{n+1}(Y) \stackrel{T}{\longrightarrow} \Omega B_{n+1}(Z) \stackrel{M}{\longrightarrow} Z, \\
& S: Z \stackrel{Q}{\longrightarrow} \Omega B_{n+1}(Z) \stackrel{U}{\longrightarrow} \Omega B_{n+1}(Y) \stackrel{N}{\longrightarrow} Y
\end{aligned}
$$

form $n$-HM maps $R$ from $Y$ to $Z$ and $S$ from $Z$ to $Y$. Since $g f: B_{n+1}(Y) \rightarrow B_{n+1}(Y)$ is homotopic to the identity, $U T$ is $(n-\mathrm{HM})$ homotopic to $\left\{I_{0}, \ldots, I_{n}\right\}$. Since $Q M$ is $(n-\mathrm{HM})$ homotopic to $\left\{I_{0}, \ldots, I_{n}\right\}$ and $N P$ is $\left(n\right.$-HM) homotopic to $\left\{I_{0}, \ldots, I_{n}\right\}$, then $S R=N U Q M T P \simeq\left\{I_{0}, \ldots, I_{n}\right\}$ where in the above formulas, $\left\{I_{0}, \ldots, I_{n}\right\}$ is the $n$-HM map induced by the identity map of the appropriate space. Similarly $R S \simeq\left\{I_{0}, \ldots, I_{n}\right\}$. Thus $Y$ and $Z$ are $n$-equivalent.

\subsubsection{Theorem. Suppose $X$ and $Y$ are $\mathrm{CW} H$-spaces. Suppose}

$$
M=\left\{M_{0}, M_{1}, \ldots, M_{i}, \ldots\right\}
$$

is an $\infty-H M$ map from $X$ to $Y$, and that $N_{0}: Y \rightarrow X$ is a homotopy inverse to $M_{0}$. Then $X$ and $Y$ are $\infty$-equivalent.

Proof. In the diagram

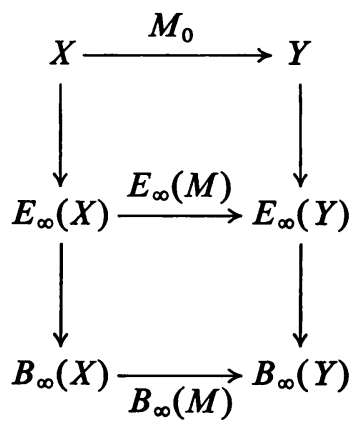


the top two horizontal maps induce isomorphisms in homology and so by the Moore Comparison Theorem [2, 3-04] the map $B_{\infty}(M)$ does also. Hence $B_{\infty}(X)$ and $B_{\infty}(Y)$ have the same homotopy type and thus $X$ and $Y$ are $\infty$-equivalent.

\section{Appendix.}

Proof of Theorem 3.5. Suppose we are given a quasifibering $F \rightarrow E \rightarrow X$, where $X$ is a $\mathrm{CW}$-complex, and we are given the homotopy commutative diagram

where

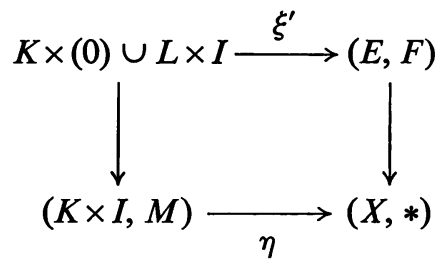

$$
\begin{aligned}
& K=\Omega X \times(I \times \Omega X)^{n}=(\Omega X)_{n}, \\
& L=K \cap\left\{\omega \mid t_{i}(\omega) \in \delta I \text { for some } i\right\}, \\
& M=K \times \delta I, \\
& M^{\prime}=M \cap K \times(0) \cup L \times I \text { and }
\end{aligned}
$$

the homotopy $y$ between $p \xi^{\prime}$ and $\eta \mid K \times 0 \cup L \times I$ satisfies $y\left(M^{\prime} \times I\right) \subset(*)$.

To construct $\xi$ and $y$, since $X$ is a CW-complex, $\Omega X$ has the homotopy type of a CW-complex $\tilde{X}$ [8]. Thus we have $X \stackrel{i}{\rightarrow} \Omega X \stackrel{j}{\rightarrow} X$ so that $j i \simeq$ id. Now define the homotopy commutative diagram as follows:

$$
\begin{aligned}
& \left(\bar{K} \times(0) \cup \bar{L} \times I, \bar{M}^{\prime}\right) \stackrel{\xi^{\prime}}{\longrightarrow}(E, F) \\
& \bar{K}=\tilde{X} \times(I \times \tilde{X})^{n}=(\tilde{X})_{n}, \quad \bar{L}=\bar{K} \cap\left\{\omega \mid t_{i}(\omega) \in \delta I \text { for some } i\right\}, \\
& \bar{M}=\bar{K} \times 2 I, \quad \bar{M}^{\prime}=\bar{M} \cap(\bar{K} \times(0) \cup \bar{L} \times I), \\
& \xi^{\prime}(\omega, s)=\xi^{\prime}(i(\omega), s), \quad \bar{\eta}(\omega, s)=\eta(i(\omega), s), \\
& \bar{y}: p \bar{\xi} \simeq \bar{\eta} \mid \bar{K} \times(0) \cup \bar{L} \times I, \quad y(\omega, s, t)=y(i(\omega), s, t) \quad \text { if } 0 \leqq t \leqq 1,
\end{aligned}
$$

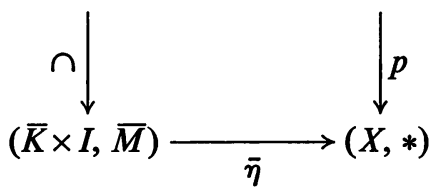

where in the above formulas, if $\omega=\left(\lambda_{0}, t_{1}, \lambda_{1}, \ldots, t_{n}, \lambda_{n}\right)$, by $i(\omega)$ we mean $i(\omega)=\left(i\left(\lambda_{0}\right), t_{1}, i\left(\lambda_{1}\right), \ldots, t_{n}, i\left(\lambda_{n}\right)\right)$. Then by the ACHEP (for CW-pairs), $\xi^{\prime}$ extends to $\xi:(\bar{K} \times I, \bar{M}) \rightarrow(E, F)$ and $\bar{y}$ extends to $\bar{Y}: p \bar{\xi} \simeq \bar{\eta}$ and $\bar{Y}(I \times \bar{M}) \subset(*)$, so we have the homotopy commutative diagram:

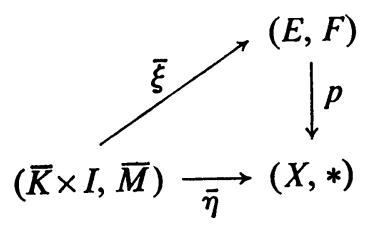


Now define $\xi^{\prime \prime}:(K \times I, M) \rightarrow(E, F)$ by $\xi^{\prime \prime}(\omega, s)=\xi(j(\omega), s)$ where if $\omega=$ $\left(\lambda_{0}, t_{1}, \lambda_{1}, \ldots, t_{n}, \lambda_{n}\right)$,

$$
j(\omega)=\left(j\left(\lambda_{0}\right), t_{1}, \ldots, t_{n}, j\left(\lambda_{n}\right)\right) .
$$

Now $\xi^{\prime \prime}$ is not an extension of $\xi^{\prime}$, but $\xi^{\prime \prime} \mid K \times(0) \cup L \times I$ is homotopic to $\xi^{\prime}$, which is all that we will need.

The geometric idea of what we will do now is this: For fixed $\lambda_{0}, \ldots, \lambda_{n}$ and $s$, we may consider $\xi^{\prime}$ as a function defined on $\partial I^{n}$. For fixed $\lambda_{0}, \ldots, \lambda_{n}$, and $s$, we may consider $\xi^{\prime \prime}$ as function defined on $I^{n}$. However $\xi^{\prime}$, considered as a function on $\partial I^{n}$, is homotopic to $\xi^{\prime \prime}$ restricted to $\partial I^{n}$, considering $\xi^{\prime \prime}$ as a function on $I^{n}$. Thus we may define $\xi$ on $I^{n}$, then enclose $I^{n}$ in a larger copy of $I^{n}$, and pass from $\xi$ to $\xi^{\prime}$ by the homotopy.

To be more precise, let $J^{n}=[-1,2]^{n}$. Let $\left(t_{1}, \ldots, t_{n}\right) \in J^{n}$. If $\left(t_{1}, \ldots, t_{n}\right) \in I^{n}$, we say the distance from $\left(t_{1}, \ldots, t_{n}\right)$ to $I^{n}$ is zero. Otherwise consider the straight line passing through the center of $I^{n},\left(\frac{1}{2}, \ldots, \frac{1}{2}\right)$, and through $\left(t_{1}, \ldots, t_{n}\right)$. The segment of this line containing $\left(t_{1}, \ldots, t_{n}\right)$ between $\partial I^{n}$ and $\partial J^{n}$ meets $\partial I^{n}$ at a point $\left(t_{1}^{1}, \ldots, t_{n}^{1}\right)$ and $\partial J^{n}$ at a point $\left(t_{1}^{2}, \ldots, t_{n}^{2}\right)$. Let $u\left(t_{1}, \ldots, t_{n}\right)$ be the ratio of the distance from $\left(t_{1}, \ldots, t_{n}\right)$ to $\left(t_{1}^{\prime}, \ldots, t_{n}^{\prime}\right)$ to the distance from $\left(t_{1}^{\prime}, \ldots, t_{n}^{\prime}\right)$ to $\left(t_{2}^{\prime}, \ldots, t_{n}^{\prime}\right)$.

Define $\xi^{\prime \prime}: \Omega X \times(J \times \Omega X)^{n} \times I \rightarrow E$ by letting, if $\omega=\left(\lambda_{0}, t_{1}, \ldots, t_{n}, \lambda_{n}\right) \in \Omega X \times$ $(J \times \Omega X)^{n}$,

$$
\begin{aligned}
\xi^{\prime \prime \prime}(\omega, s) & =\xi^{\prime}(\omega, s) \quad \text { if } t=\left(t_{1}, \ldots, t_{n}\right) \in I^{n}, \\
& =\xi^{\prime}\left(h_{u(t)}\left(\lambda_{0}\right), t_{1}^{1}, h_{u(t)}\left(\lambda_{1}\right), \ldots, t_{n}^{1}, h_{u(t)}\left(\lambda_{n}\right), s\right) \quad \text { if } t \in J^{n}-I^{n},
\end{aligned}
$$

where in the above formula, $h$ is the homotopy between $i j$ and id. That is, $h_{0}=i j$ and $h_{1}=\mathrm{id}$. We note that if $t_{i} \in \partial I$ for some $i$, then

$$
\begin{aligned}
\xi^{\prime \prime}(\omega, s) & =\xi(j(\omega), s)=\xi^{\prime}(j(\omega), s)=\xi^{\prime}(i(j(\omega)), s) \\
& =\xi^{\prime}\left(h_{u(t)}\left(\lambda_{0}\right), t_{1}^{1}, \ldots, t_{n}^{1}, h_{u(t)}\left(\lambda_{n}\right), s\right)
\end{aligned}
$$

so that these two functions are patched together correctly.

Now consider the homeomorphism stretching $I^{n}$ onto $J^{n}$ along radial lines, sending $t=\left(t_{1}, \ldots, t_{n}\right)$ into $v=\left(v_{1}, \ldots, v_{n}\right) \in J^{n}$ so that in particular if $t \in \partial I^{n}$ then $v \in \partial J^{n}$ and $v^{1}=\left(v_{1}^{1}, \ldots, v_{n}^{1}\right)=t$. Using this mapping, we now define $\xi: \Omega X \times$ $(I \times \Omega X)^{n} \times I=K \times I \rightarrow E$ by

$$
\xi\left(\lambda_{0}, t_{1}, \ldots, t_{n}, \lambda_{n}, s\right)=\xi^{\prime \prime \prime}\left(\lambda_{0}, v_{1}, \lambda_{1}, \ldots, \lambda_{n}, s\right) .
$$

To see that $\xi$ is an extension of $\xi^{\prime}$, suppose $t_{i} \in \partial I$ for some $i$. Then $v \in \partial J^{n}$ and $t=v^{1}$ and $u(v)=1$, and

$$
\begin{aligned}
\xi\left(\lambda_{0}, t_{1}, \ldots, t_{n}, \lambda_{n}, s\right) & =\xi^{\prime \prime}\left(\lambda_{0}, v_{1}, \lambda_{1}, \ldots, v_{n}, \lambda_{n}, s\right) \\
& =\xi^{\prime}\left(h_{u(v)}\left(\lambda_{0}\right), v_{1}^{1}, h_{u(v)}\left(\lambda_{1}\right), \ldots, v_{n}^{1}, h_{u(v)}\left(\lambda_{1}\right)\right) \\
& =\xi^{\prime}\left(\lambda_{0}, t_{1}, \ldots, t_{n}, \lambda_{n}, s\right) .
\end{aligned}
$$

If $(\omega, s) \in M$, then $s \in \partial I$ and by tracing through the various stages of the construction of $\xi$, we see that $\xi(\omega, s) \in F$. 
To finish the proof, we need to construct a homotopy $Y:(K \times I \times I, M \times I) \rightarrow$ $(X, *)$ satisfying

$$
\begin{aligned}
Y(\omega, s, z) & =y(\omega, s, 2 z) \text { if } 0 \leqq z \leqq \frac{1}{2} \text { if }(\omega, s) \in K \times 0 \cup L \times I, \\
& =\eta(\omega, s) \text { if } \frac{1}{2} \leqq s \leqq 1 .
\end{aligned}
$$

This seems reasonable, since $p \xi$ is defined in terms of $p \xi^{\prime \prime}$ and $p \xi^{\prime}$, both of which are homotopic to $\eta$.

To begin the construction, we write down the formulas for $p \xi$. Suppose $\omega=\left(\lambda_{0}, t_{1}, \ldots, t_{n}, \lambda_{n}\right) \in K$. Then

$$
\begin{aligned}
p \xi(\omega, s) & =p \xi^{\prime \prime}\left(\lambda_{0}, v_{1}, \ldots, v_{n}, \lambda_{n}, s\right) \quad \text { if } v=\left(v_{1}, \ldots, v_{n}\right) \in I^{n} \\
& =p \xi^{\prime}\left(h_{u(v)}\left(\lambda_{0}\right), v_{1}^{1}, h_{u(v)}\left(\lambda_{1}\right), \ldots, h_{u(v)}\left(\lambda_{n}\right), s\right) \text { if } v \in J^{n}-I^{n} \\
& =p \xi\left(j\left(\lambda_{0}\right), v_{1}, j\left(\lambda_{1}\right), \ldots, v_{n}, j\left(\lambda_{n}\right), s\right) \text { if } v \in I^{n} \\
& =p \xi^{\prime}\left(h_{u(v)}\left(\lambda_{0}\right), v_{1}^{1}, \ldots, h_{u(v)}\left(\lambda_{n}\right), s\right) \text { if } v \in J^{n}-I^{n} \\
& =\bar{Y}\left(j\left(\lambda_{0}\right), v_{1}, \ldots, v_{n}, j\left(\lambda_{n}\right), s, 0\right) \text { if } v \in I^{n} \\
& =y\left(h_{u(v)}\left(\lambda_{0}\right), v_{1}^{1}, \ldots, v_{n}^{1}, h_{u(v)}\left(\lambda_{n}\right), s, 0\right) \text { if } v \in J^{n}-I^{n}
\end{aligned}
$$

So we let $W^{1}$ be a homotopy defined as follows: If $\omega=\left(\lambda_{0}, t_{1}, \ldots, t_{n}, \lambda_{n}\right) \in K$, $s \in I$, and $z \in I$

$$
\begin{aligned}
W^{1}(\omega, s, z) & =\bar{Y}\left(j\left(\lambda_{0}\right), v_{1}, \ldots, v_{n}, j\left(\lambda_{n}\right), s, z\right) \quad \text { if } v \in I^{n} \\
& =y\left(h_{u(v)}\left(\lambda_{0}\right), v_{1}^{1}, \ldots, h_{u(v)}\left(\lambda_{n}\right), s, z\right) \quad \text { if } v \in J^{n}-I^{n}
\end{aligned}
$$

To see that $W^{1}$ is well defined, if $v \in \partial I^{n}$ then $v=v^{1}$ and $u(v)=0$, so

$$
\begin{aligned}
\bar{Y}\left(j\left(\lambda_{0}\right), v_{1}, \ldots, v_{n}, j\left(\lambda_{n}\right), s, z\right) & =\bar{y}\left(j\left(\lambda_{0}\right), v_{1}, \ldots, v_{n}, j\left(\lambda_{n}\right), s, z\right) \\
& =Y\left(i j\left(\lambda_{0}\right), v_{1}, \ldots, v_{n}, s, z\right) \\
& =y\left(h_{u(v)}\left(\lambda_{0}\right), v_{1}^{1}, \ldots, v_{n}^{1}, h_{u(v)}\left(\lambda_{n}\right), s, z\right) .
\end{aligned}
$$

Thus $W^{1}$ gives a homotopy between $p \xi$ and the function $\tau$ defined as follows: Let $\omega=\left(\lambda_{0}, t_{1}, \ldots, t_{n}, \lambda_{n}\right) \in K$.

$$
\begin{aligned}
\tau(\omega, s) & =\bar{\eta}\left(j\left(\lambda_{0}\right), v_{1}, \ldots, v_{n}, j\left(\lambda_{n}\right), s\right) \quad \text { if } v \in I^{n}, \\
& =\eta\left(h_{u(v)}\left(\lambda_{0}\right), v_{1}^{1}, \ldots, v_{n}^{1}, h_{u(v)}\left(\lambda_{n}\right), s\right) \quad \text { if } v \in J^{n}-I^{n} .
\end{aligned}
$$

Then by the definitions of $\bar{\eta}$ and $h$ we have

$$
\begin{aligned}
\tau(\omega, s) & =\eta\left(i j\left(\lambda_{0}\right), v_{1}, \ldots, v_{n}, i j\left(\lambda_{n}\right), s\right) \text { if } v \in I^{n} \\
& =\eta\left(h_{u(v)}\left(\lambda_{0}\right), v_{1}^{1}, \ldots, v_{n}^{1}, h_{u(v)}\left(\lambda_{n}\right), s\right) \text { if } v \in J^{n}-I^{n}, \\
& =\eta\left(i j\left(\lambda_{0}\right), v_{1}^{1}, \ldots, v_{n}^{1}, h_{u(v)}\left(\lambda_{n}\right), s\right) \text { if } v \in I^{n} \\
& =\eta\left(h_{u(v)}\left(\lambda_{0}\right), v_{1}^{1}, \ldots, v_{n}^{1}, h_{u(v)}\left(\lambda_{n}\right), s\right) \text { if } v \in J^{n}-I^{n}
\end{aligned}
$$

Then we define a homotopy $W^{2}$ between $\tau$ and $\eta$ as follows: Let

$s \in I$, and $z \in J$.

$$
\omega=\left(\lambda_{0}, t_{1}, \ldots, t_{n}, \lambda_{n}\right) \in K,
$$

$$
\begin{aligned}
W^{2}(\omega, s, z) & =\eta\left(h_{0}\left(\lambda_{0}\right), v_{1}, \ldots, v_{n}, h_{z}\left(\lambda_{n}\right), s\right) \quad \text { if } v \in I^{n} \\
& =\eta\left(h_{B(z)}\left(\lambda_{0}\right), v_{1}^{1}, \ldots, h_{\beta(z)}\left(\lambda_{n}\right), s\right) \quad \text { if } v \in J^{n}-I^{n}
\end{aligned}
$$


In the above formula, $\beta(z)=u(v)+z(1-u(v))$.

Then finally we define the homotopy $Y$ by

$$
\begin{aligned}
Y(\omega, s, z) & =W^{1}(\omega, s, 2 z) \text { if } 0 \leqq z \leqq \frac{1}{2} \\
& =W^{2}(\omega, s, 2 z-1) \text { if } \frac{1}{2} \leqq z \leqq 1
\end{aligned}
$$

Then $Y:(K \times I \times I, M \times I) \subset(X, *)$ satisfies $Y(, 0)=p \xi$ and $Y(, 1)=\eta$. Now suppose $(\omega, s) \in K \times(0) \cup L \times I$. Then, if $\omega=\left(\lambda_{0}, t_{1}, \ldots, t_{n}, \lambda_{n}\right)$,

$$
\begin{aligned}
Y(\omega, s, z)=W^{1}(\omega, s, 2 z) & =y\left(\lambda_{0}, v_{1}^{1}, \lambda_{1}, \ldots, v_{n}^{1}, \lambda_{n}, s, 2 z\right) \\
& =y(\omega, s, 2 z) \text { if } 0 \leqq z \leqq \frac{1}{2} \\
Y(\omega, s, z)=W^{2}(\omega, s, 2 z-1) & =\eta\left(h_{\beta(2 z-1)}\left(\lambda_{0}\right), v_{1}^{1}, \ldots, v_{n}^{1}, h_{\beta(2 z-1)}\left(\lambda_{n}\right), s\right) \\
& =\eta\left(\lambda_{0}, v_{1}^{1}, \ldots, v_{n}^{1}, \lambda_{n}, s\right) \\
& =\eta\left(\lambda_{0}, t_{1}, \ldots, t_{n}, \lambda_{n}, s\right) \text { if } \frac{1}{2} \leqq z \leqq 1
\end{aligned}
$$

Thus

$$
\begin{aligned}
Y(\omega, s, z) & =y(\omega, s, 2 z) \quad \text { if } 0 \leqq z \leqq \frac{1}{2} \\
& =\eta(\omega, s) \text { if } \frac{1}{2} \leqq z \leqq 1
\end{aligned}
$$

\section{BIBLIOGRAPHY}

1. E. H. Brown, Jr., Twisted tensor products. I, Ann. of Math. (2) 69 (1959), 223-246. MR 21 $\# 4423$.

2. H. Cartan et al., Algèbres d'Eilenberg-Mac Lane et homotopie, Séminaire Henri Cartan, 1954/55, Secrétariat mathématique, Paris, 1956. MR 19, 439.

3. A. Clark, Homotopy commutativity and the Moore spectral sequence, Pacific J. Math. 15 (1965), 65-74. MR 31 \#1679.

4. M. L. Curtis and R. Lashof, Homotopy equivalence of fiber bundles, Proc. Amer. Math. Soc. 9 (1958), 178-182. MR 20 \#4838.

5. A. Dold and R. Lashof, Principal quasi-fibrations and fibre homotopy equivalence of bundles, Illinois J. Math. 3 (1959), 285-305. MR 21 \#331.

6. A. Dold and R. Thom, Quasifaserungen und Unendliche Symmetrische Produkte, Ann. of Math. (2) 67 (1958), 239-281. MR 20 \#3542.

7. R. J. Milgram, The Bar construction and Abelian H-spaces, Princeton Univ., Princeton, N. J. (mimeographed notes).

8. J. Milnor, Construction of universal bundles. I, II, Ann. of Math. (2) 63 (1956), 272-284, 430-436. MR 17, 994. MR 17, 1120.

9. - On spaces having the homotopy type of $C W$-complex, Trans. Amer. Math. Soc. 90 (1959), 272-280. MR 20 \#6700.

10. J. Stasheff, Homotopy associativity of H-spaces. I, Trans. Amer. Math. Soc. 108 (1963), 275-292. MR 28 \#1623.

11. - On homotopy Abelian H-spaces, Proc. Cambridge Philos. Soc. 57 (1961), 734 745. MR 25 \#4534.

12. - " "Parallel" transport in fibre spaces, Bol. Soc. Mat. Mexicana (2) 11 (1966), 68-84. MR 38 \#5219.

13. - Homotopy associativity of H-spaces. II, Trans. Amer. Math. Soc. 108 (1963), 293-312. MR 28 \#1623.

14. N. Steenrod, The topology of fibre bundles, Princeton Math. Series, vol. 14, Princeton Univ. Press, Princeton, N. J., 1951. MR 12, 522. 
15. M. Sugawara, On a condition that a space is an $\mathrm{H}$-space, Math. J. Okayama Univ. 6 (1957), 109-129. MR 19, 160.

16. - On the homotopy-commutativity of groups and loop spaces, Mem. Coll. Sci. Univ. Kyoto Ser. A Math. 33 (1960/61), 257-269. MR 22 \#11394.

17. M. Fuchs, Verallgemeinerte Homotopie-Homomorphismen und klassifizierende Räume, Math. Ann. 161 (1965), 197-230. MR 33 \#3295.

Haverford College,

Haverford, Pennsylvania 19041 